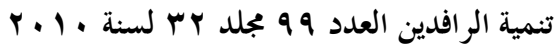

$$
\begin{aligned}
& \text { كلية الإدارة والاقتصاد -جامعة الموصل } \\
& \text { ص ص[י] }
\end{aligned}
$$

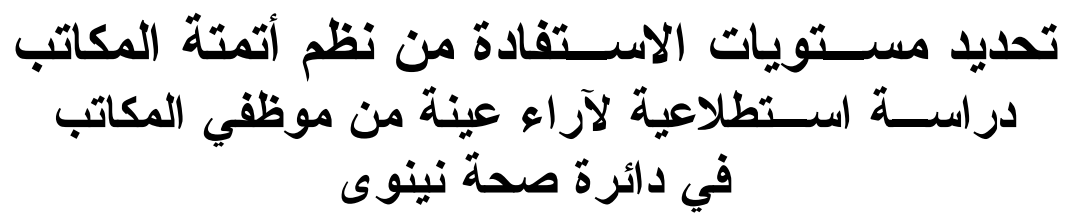

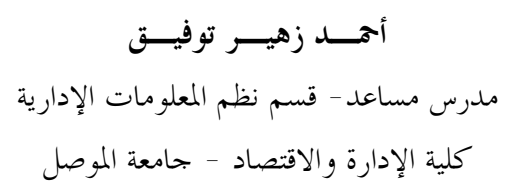

$$
\begin{aligned}
& \text { ahmedzuher76@yahoo.com" }
\end{aligned}
$$

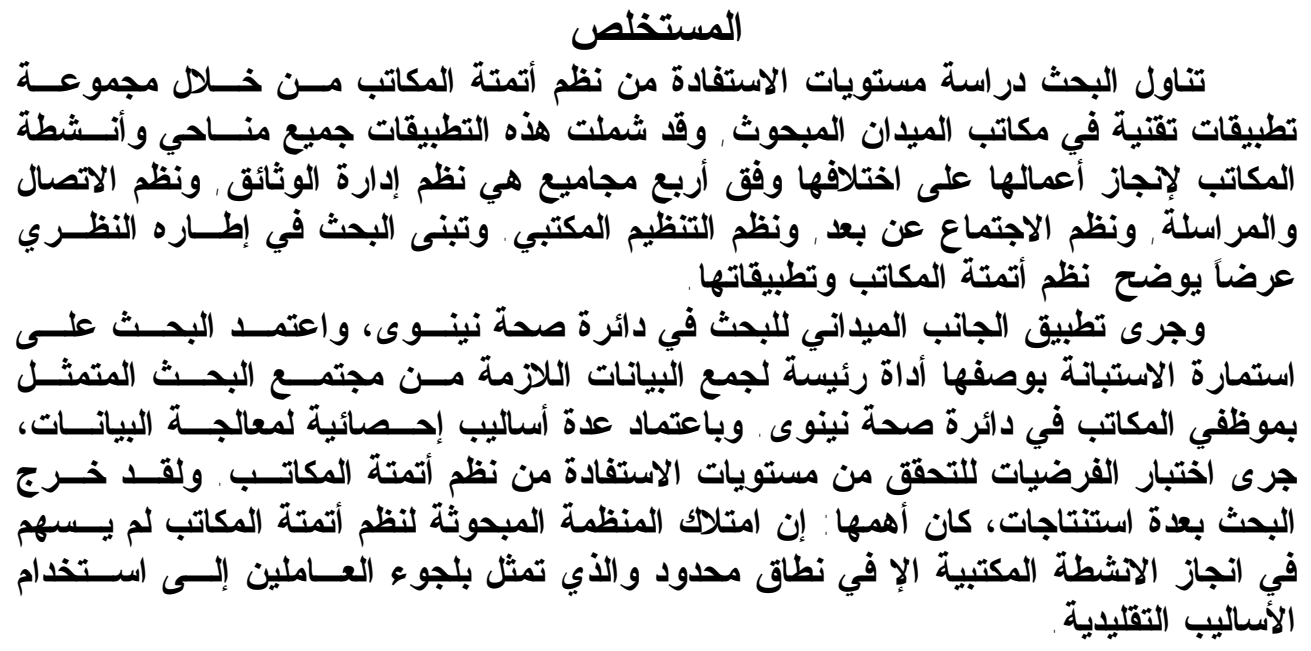

\title{
Specifying the Utility Levels of Offices Automation Systems
}

\author{
Ahmed Z. Tawfeeq \\ Assistant Lecturer \\ Department of Information Management Systems \\ University of Mosul
}

\begin{abstract}
This paper tackles the utility levels of offices automation systems through adopting a number of technical applications. These applications encompassed all the aspects and activities of such offices usually adopted to fulfill their duties on the bases of four groups: documents management systems, correspondence and communication systems, remote social systems and office organization systems. In its theoretical part, this research

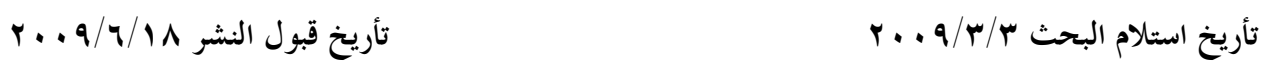




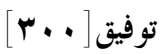

represents an illustration of the offices automation systems and their applications. Nineavah health quarter is selected as a sample and a questionnaire is adopted as a major tool for collecting the required data from the offices employees in the quarter mentioned above. A number of statistical methods are utilized to process these data in order to reach the results and testing the hypothesis to check the utility levels of offices automation systems.

المقدمة

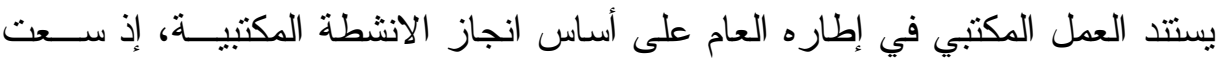

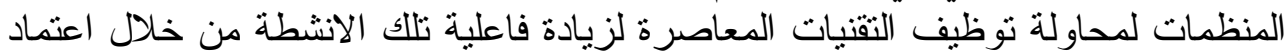

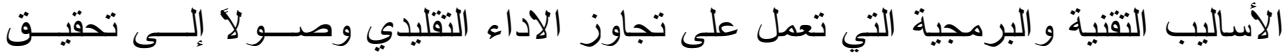

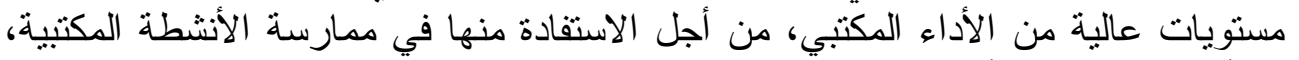

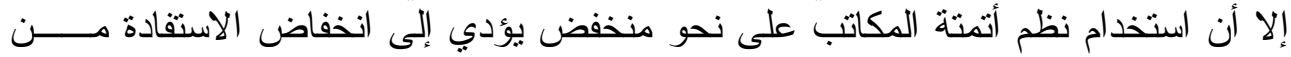

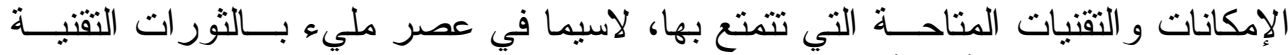

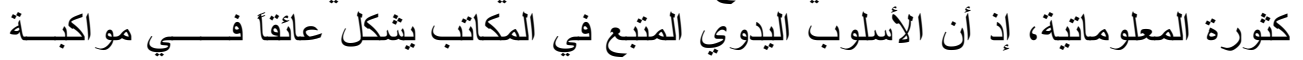
التطور، لذلك لا بُد من إدخال تقنيات الحاسوب و البرمجيات لغرض تسهيل العمل في هذه

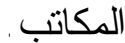

تأسيسأ على ماتقدم تضمن البحث أربعة محاور، حيث تناول المحور الأول منهجيـــة

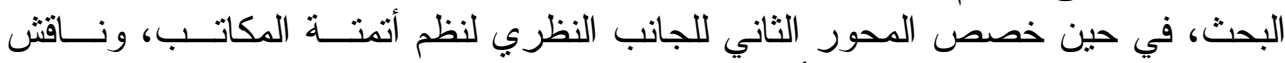
المحور الثالث الجانب الميداني، وأخير أنتاول المحور الر ابع الاستتناجات و التوصيات .

\section{منهجية البحث مشكلة البحث}

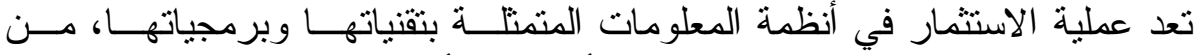

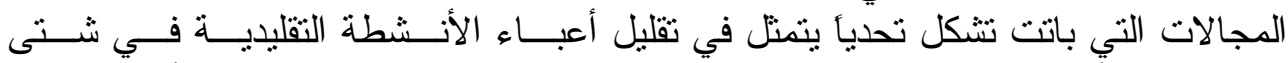

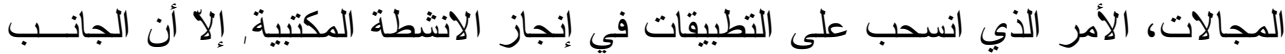

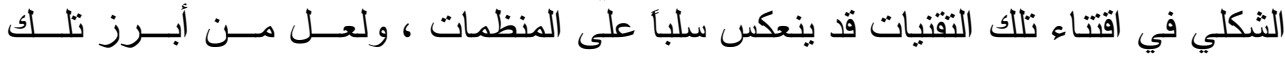

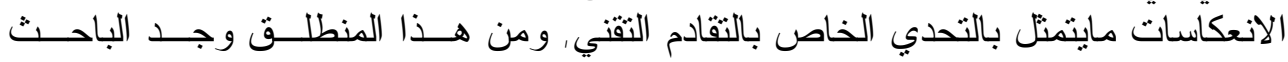

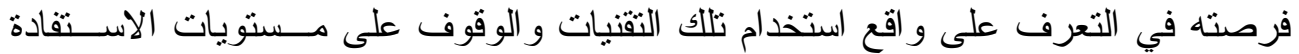
الفعلية بوصفها أبرز أوجه الاستثمار , ولتحديد المشكلة البحثية بشكل أكثر وضوحاً يمكـن

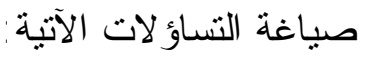
ا ـ ما مدى استخدام نظم أتمتة المكاتب في الميدان المبحة المبحوث؟

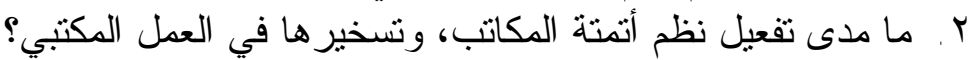
r. ما هي مستويات الاستفادة من نظم أتمتة المكاتب في المنظمة فئن المبحوثة؟

$$
\begin{aligned}
& \text { فرضيات البحث } \\
& \text { سعياً لحل مشكلة البحث تم وضع الفرضيات الآتية: }
\end{aligned}
$$

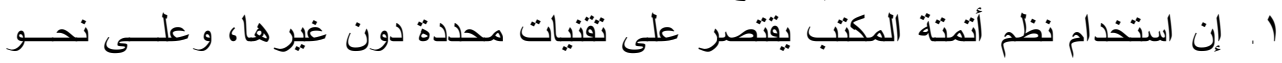

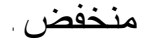

$$
\begin{aligned}
& \text { r . يقتصر تفعيل نظم أتمتة المكاتب على بعض الأنشطة المكتبية دون غير ها. }
\end{aligned}
$$




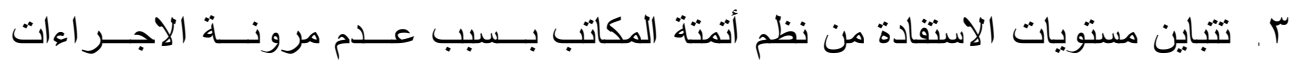

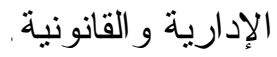

\section{أهمية البحث وأهدافه}

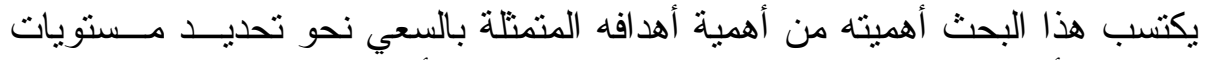

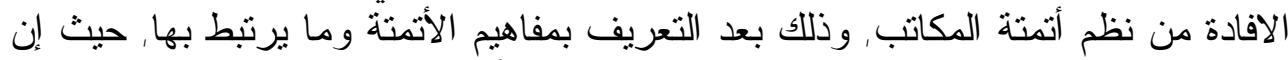

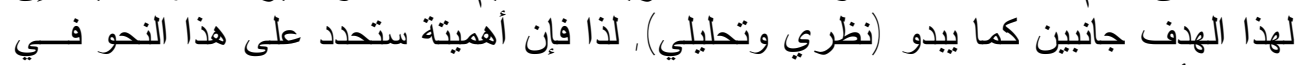

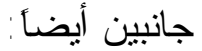

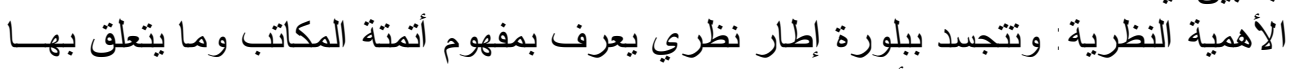

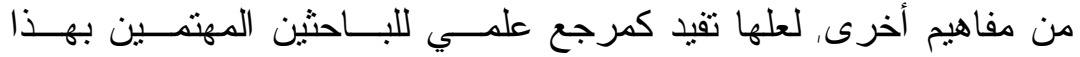

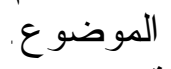

الأهمية الميدانية: وتتجسد بالسعي نحو ترجمة الهذف الششار اليه كوقائع ميدانية, للوقوف

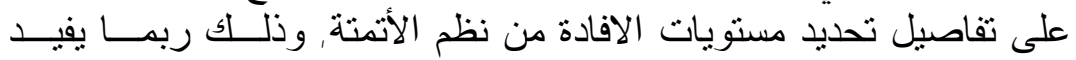

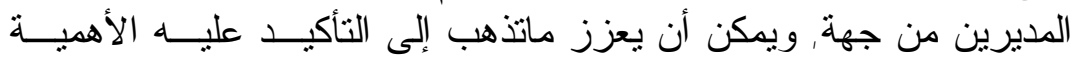

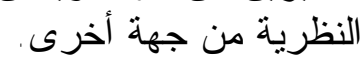

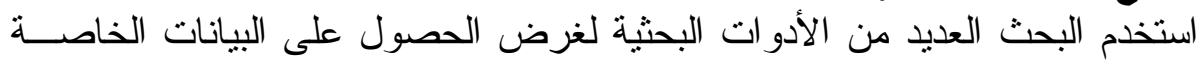

\section{أسلوب جمع البيانات وتحليلها}

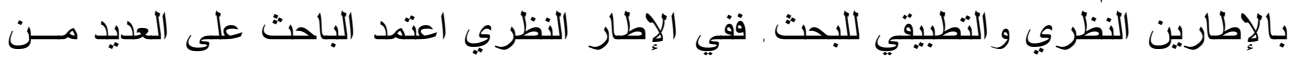

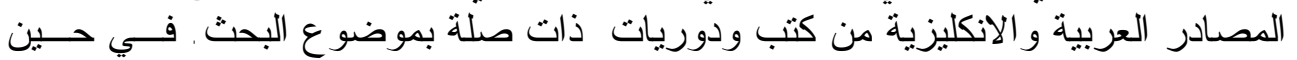

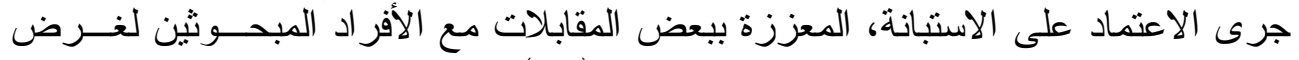

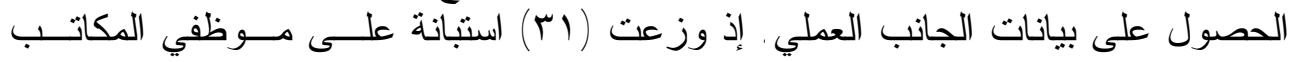

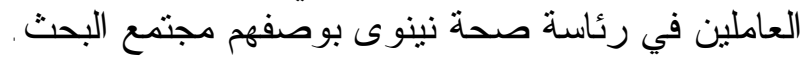

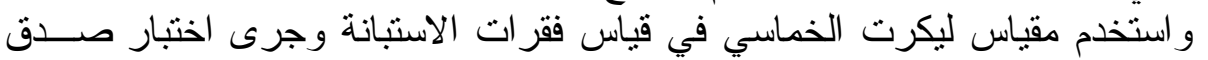

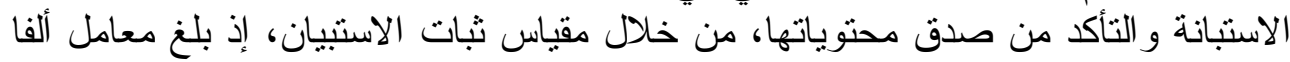

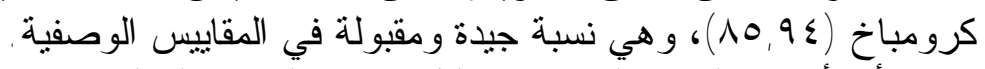

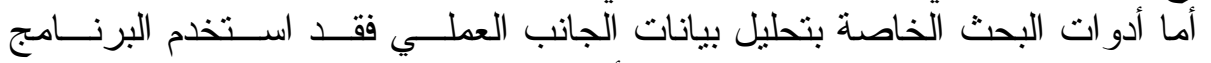

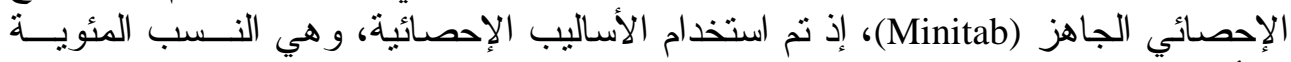
و الأوساط الحسابية و الانحر افات المعيارية.

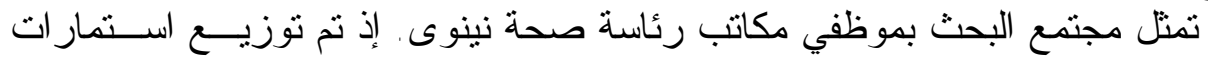

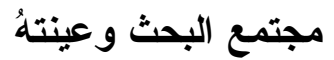

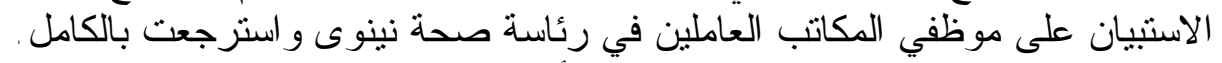

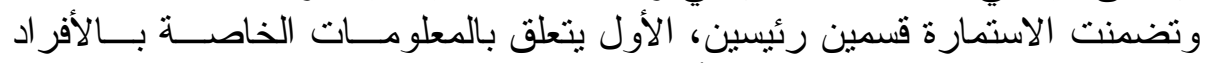

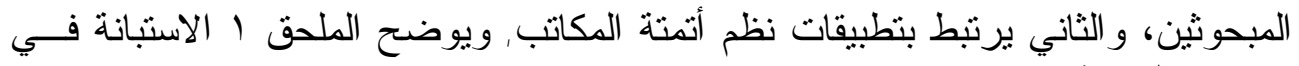

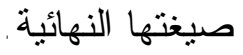




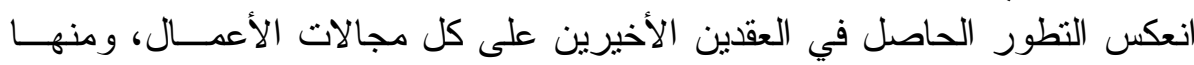
ثانياً -الجانب النظري مفهوم أتمتة المكاتب النب النب

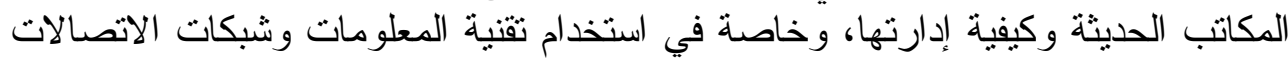

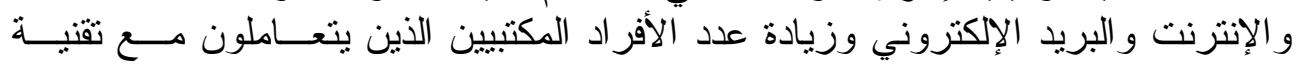

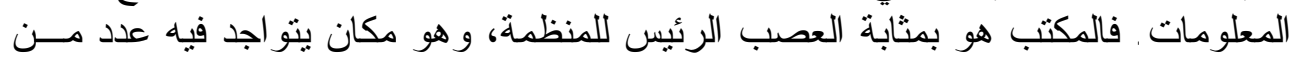

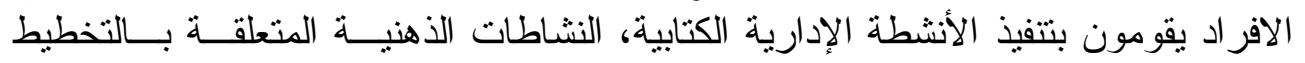

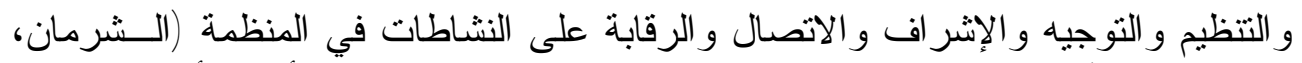

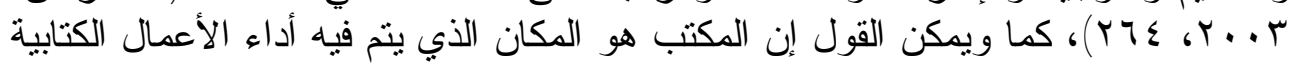

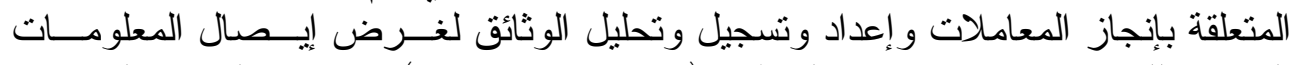

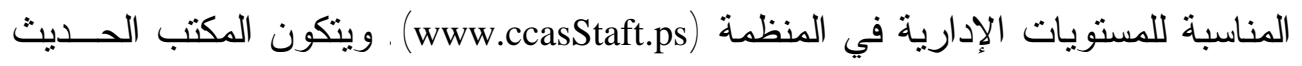

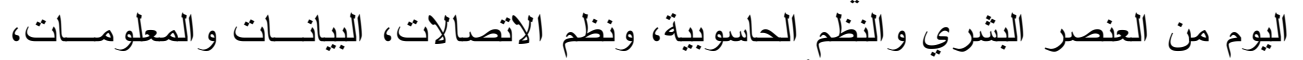

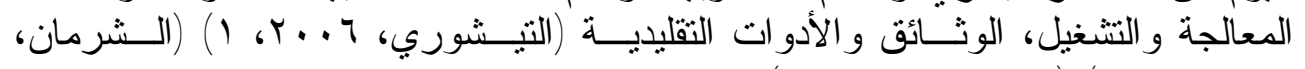

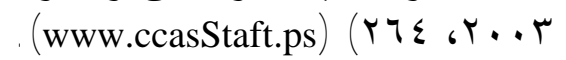

أما الأتمتة فهي التشغيل الآلي لجهاز أو عملية أو نظام يتم التحكم به آلياً بو اســـة الإسة

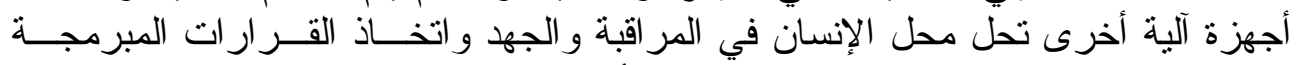

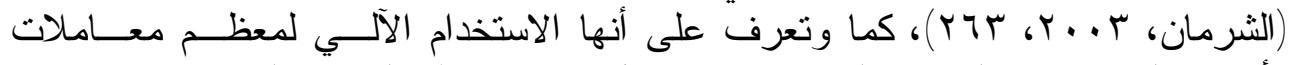

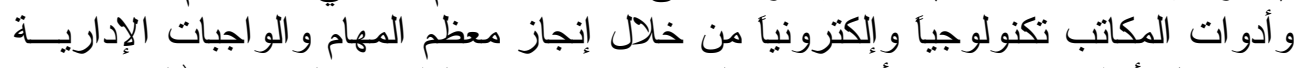

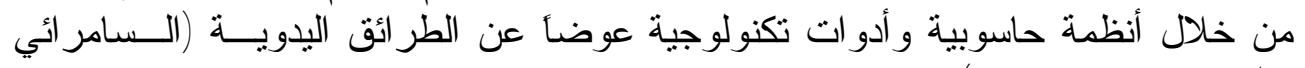

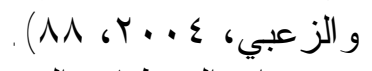

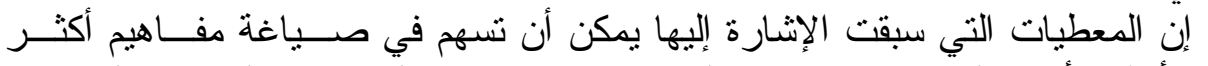

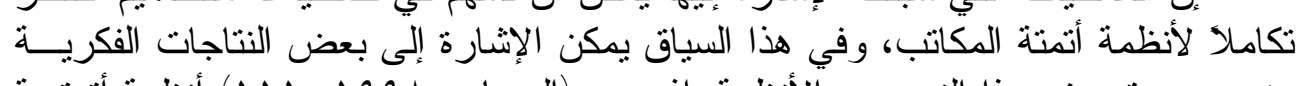

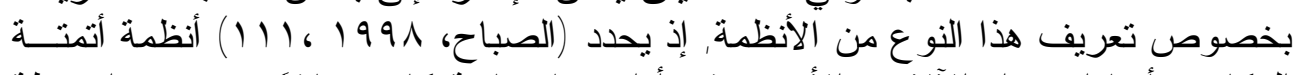

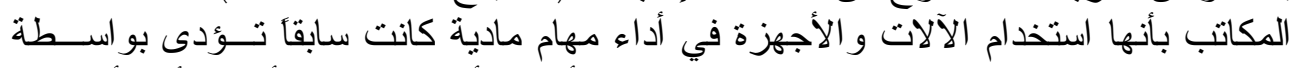

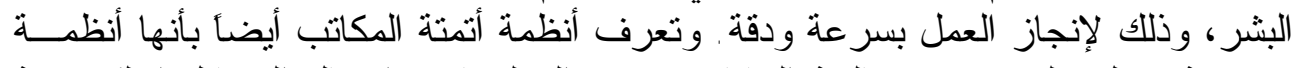

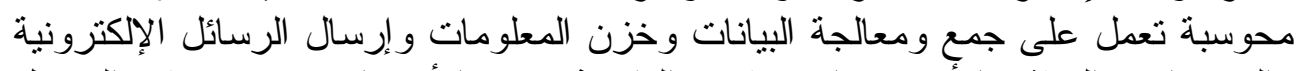

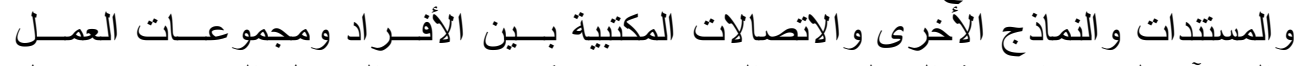

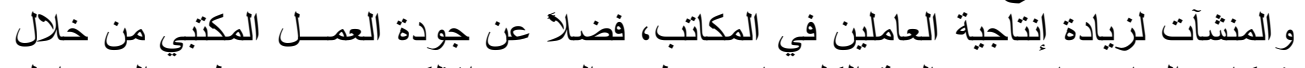

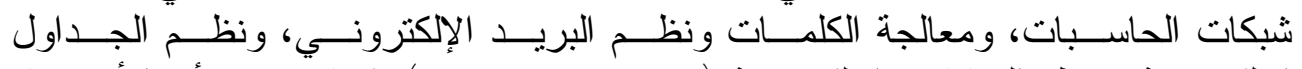

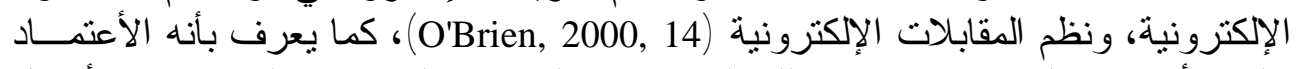

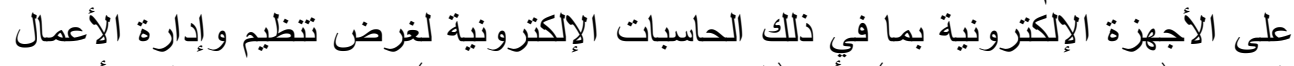

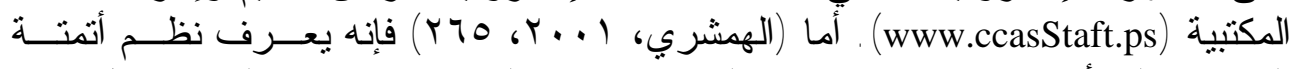

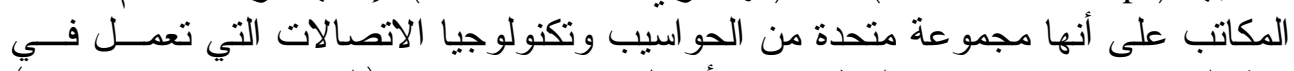

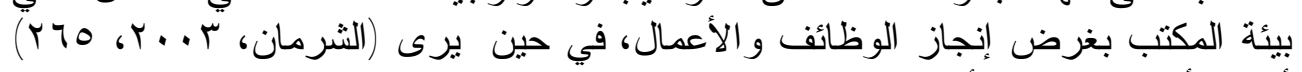

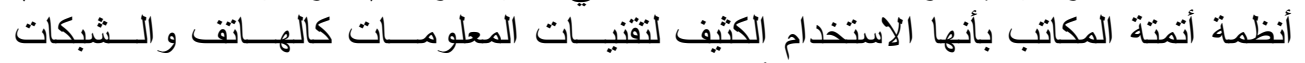

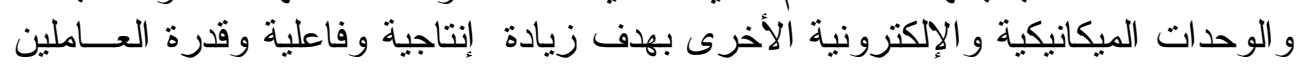

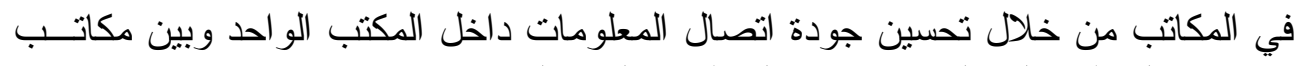
و إدار ات المنظمة المختلفة، ومكاتب المنظمة و البيئة الخارجية. 
فو ائد أنظمة أتمتة المكاتب

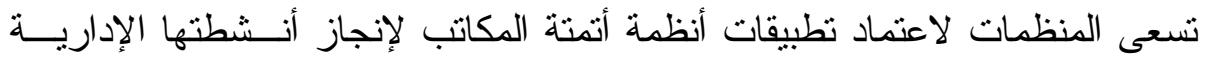

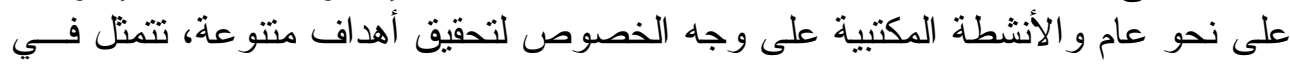

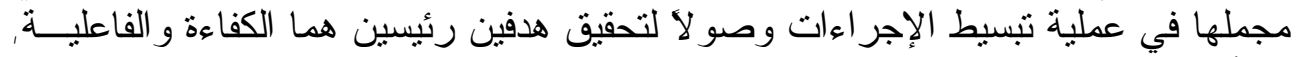

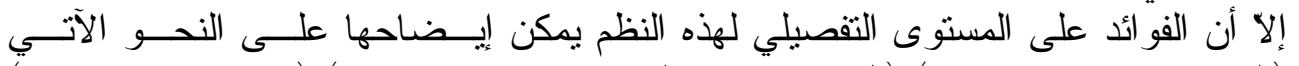

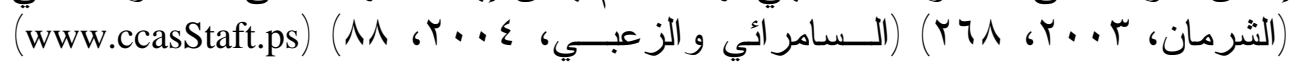

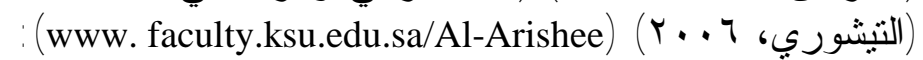

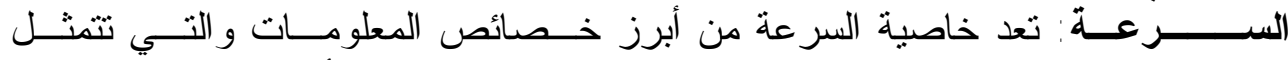

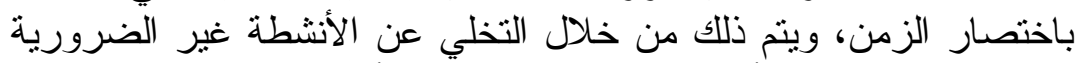

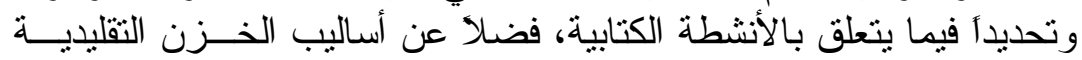

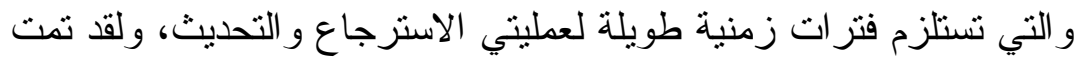

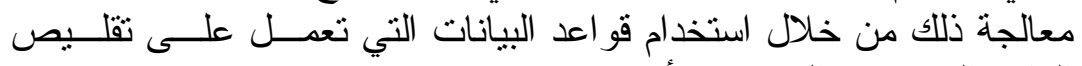

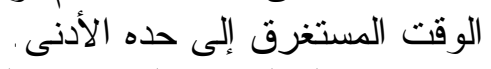

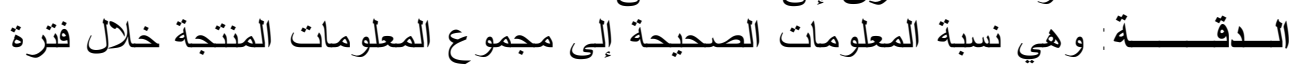

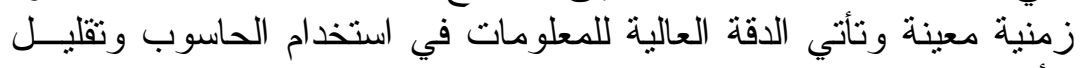

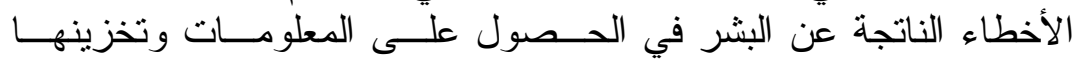

$$
\text { و استرجاعها. }
$$

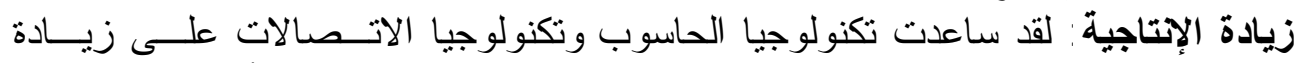

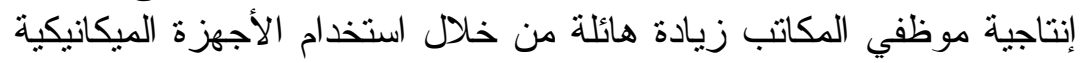

$$
\text { و الكهربائية و الإلكترونية. }
$$

الفــاعليــــــة: المتمتلة في تحسين نوعية الإكئنة الانجاز الإداري وزيادة الجودة.

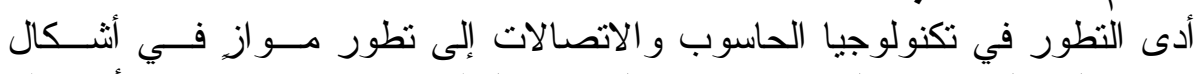

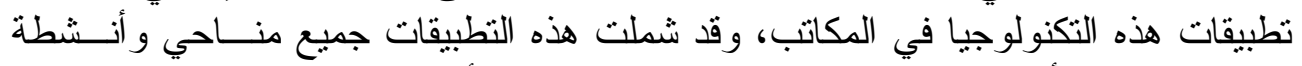

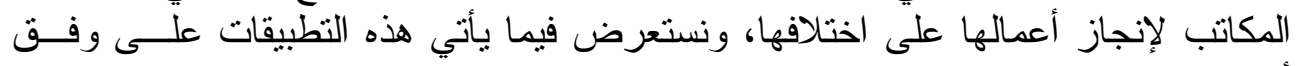

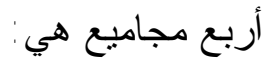

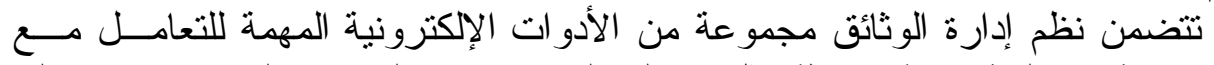

أ. نظم إدارة الوثائق

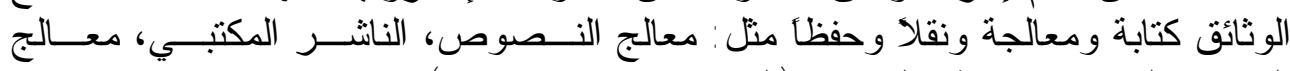

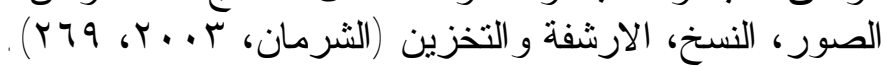

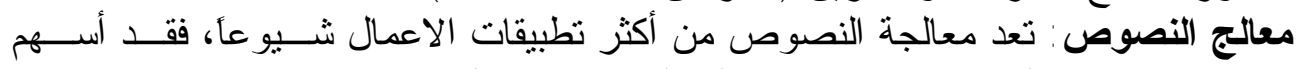

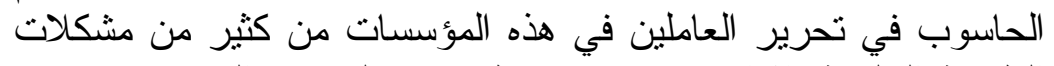

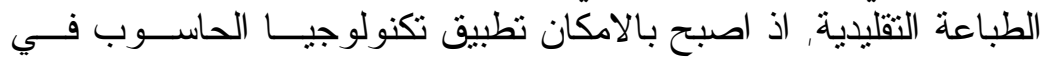

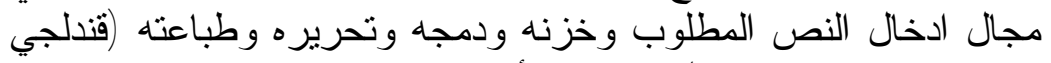

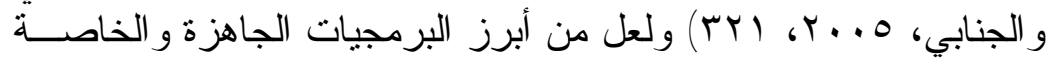

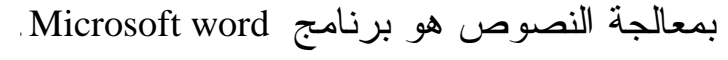




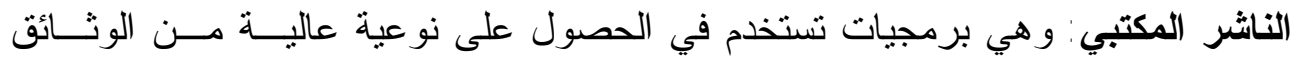

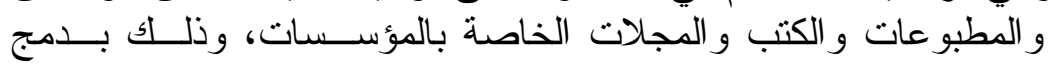

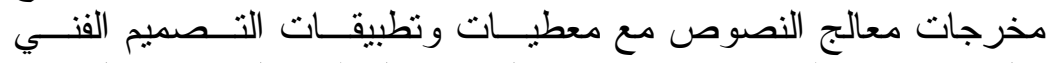

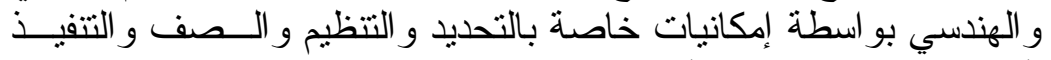

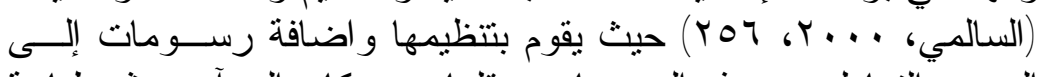

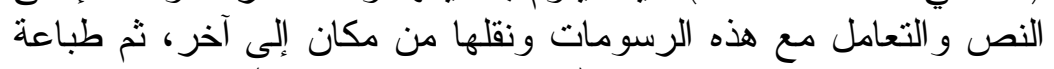

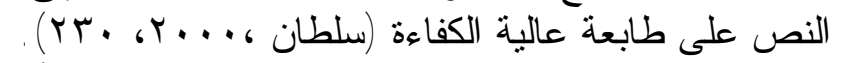

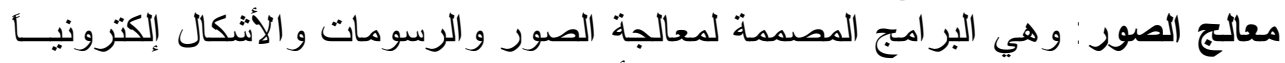

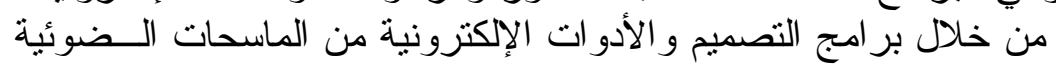

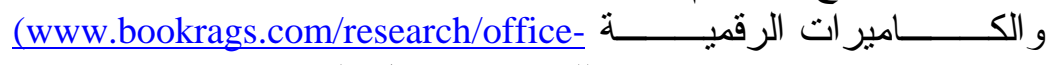
إلها automation-systems-)

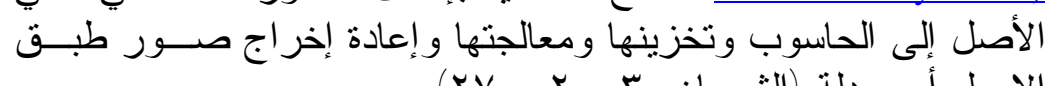

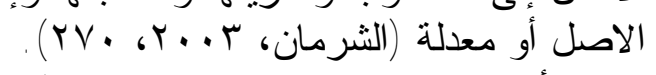

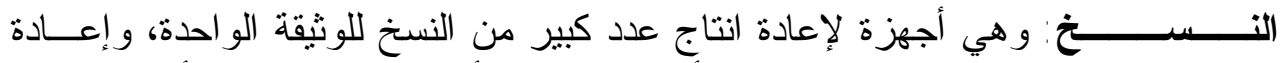

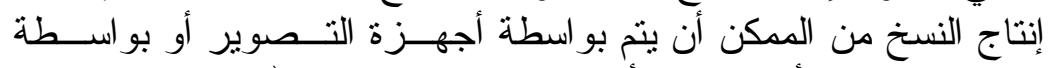

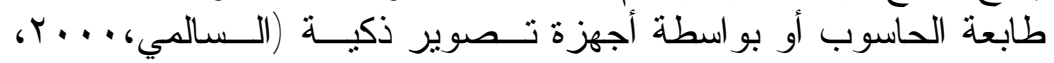
. (109

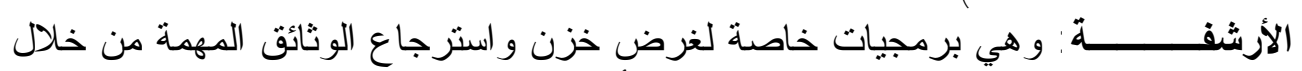

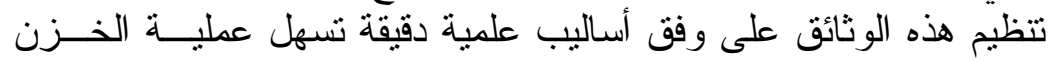

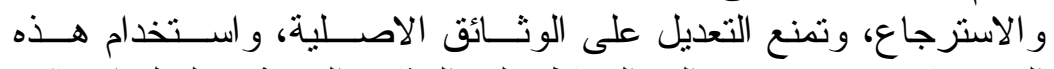

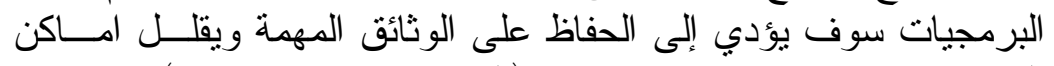

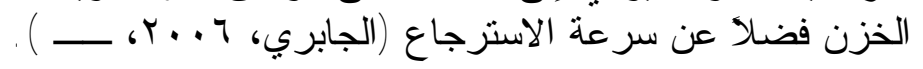

ب. نظم الاتصال والمراسلة: وتشمل الاتصالات الإكترونية منـلـل البريـــــ الإلكترونــي

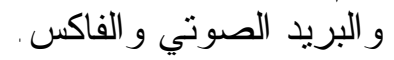

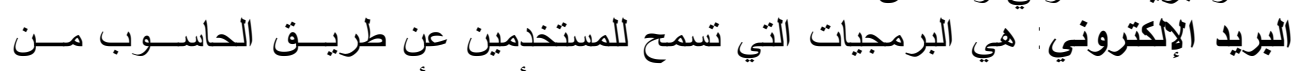

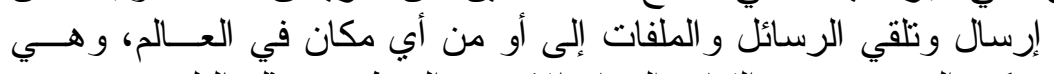

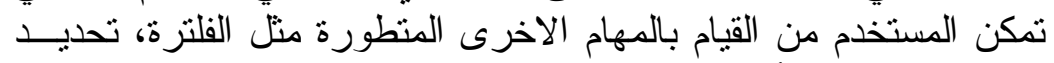

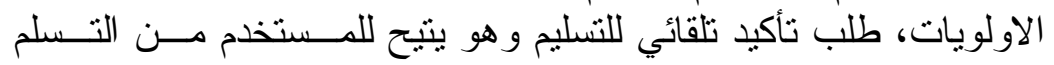

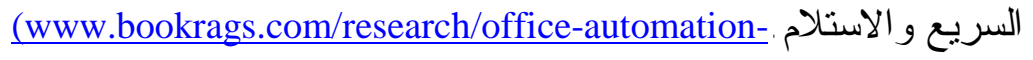
. systems-)

البريد الصوتي: يشبه البريد الصوتي البريد الإلكتروني لكنه يستخدم الصوت لنقل الرسائل

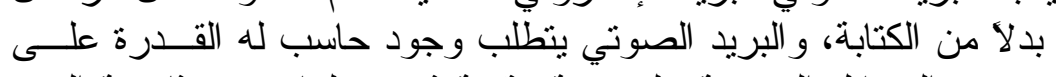

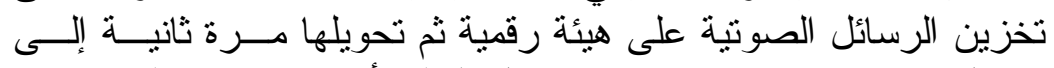

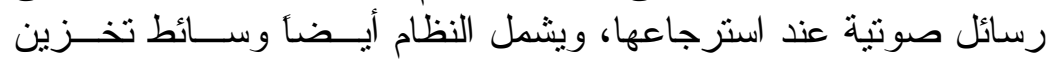

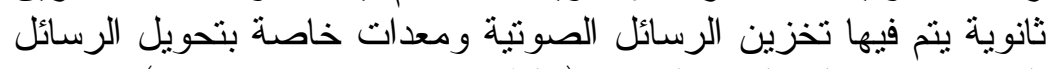

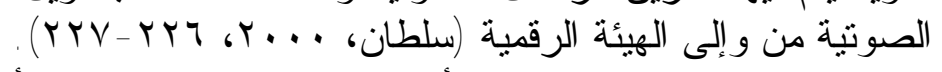

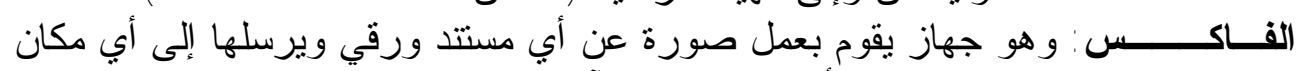

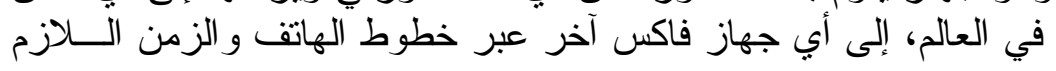


هو الزمن المستغرق نفسه لعمل صورة عن أي مستتد باستخدام جهــاز

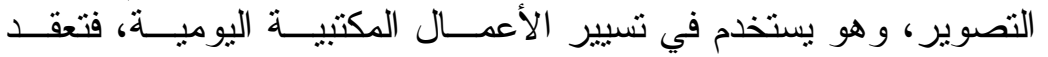

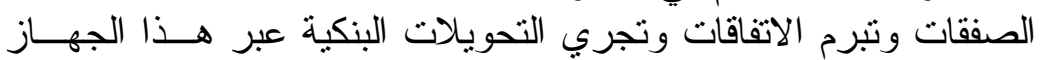
.(www.hazemsakeek.com)

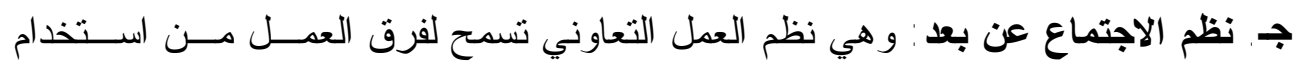

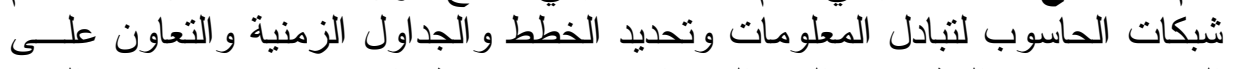

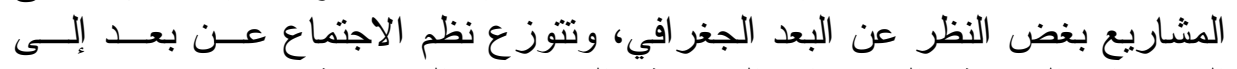

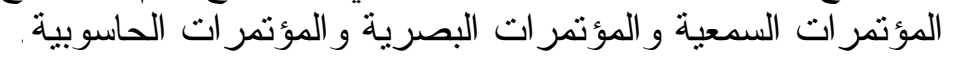

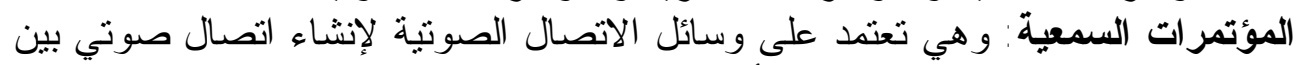

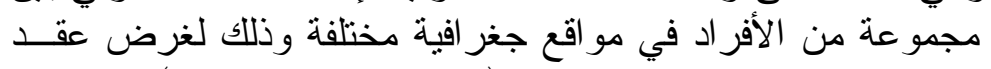

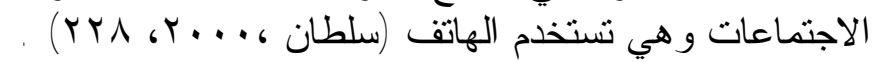

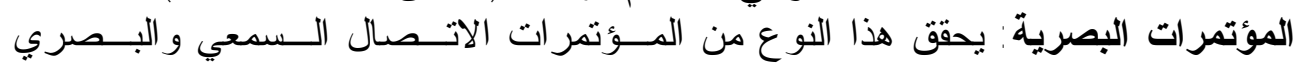

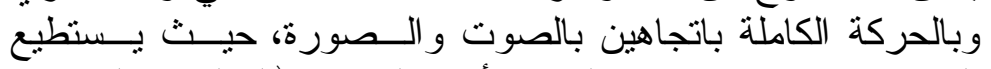

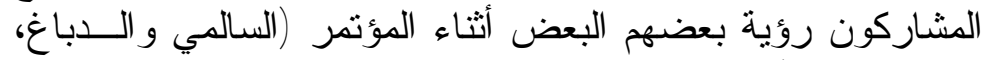

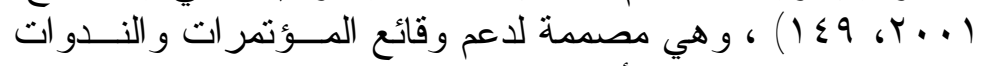

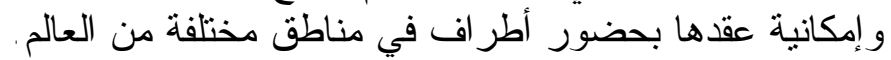

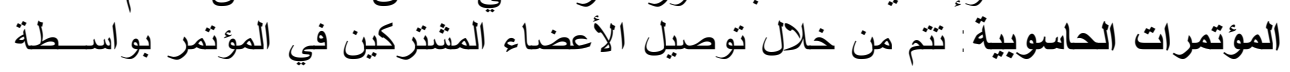

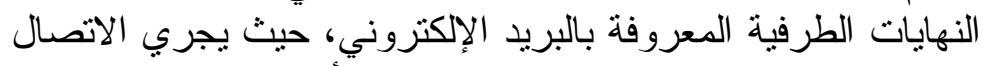

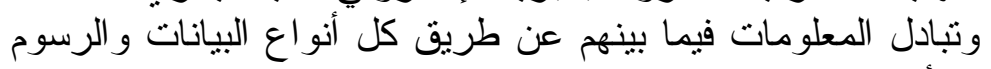

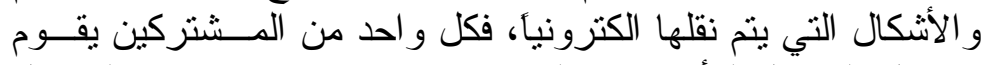

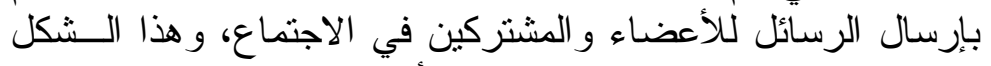

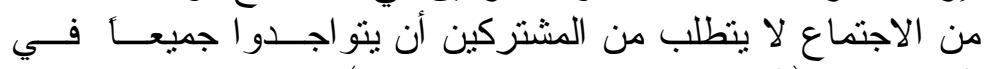

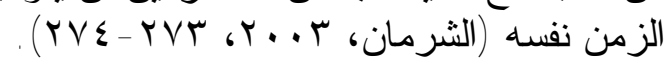

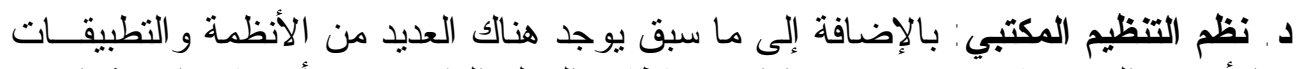

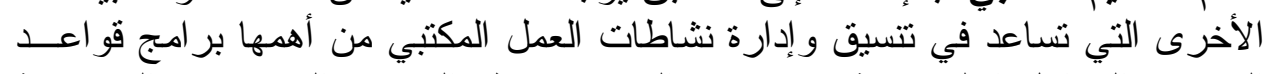

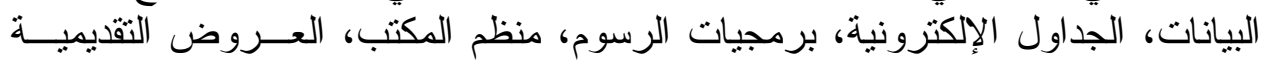

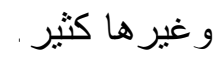

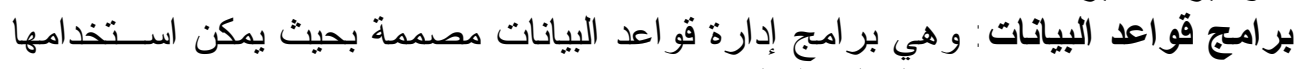

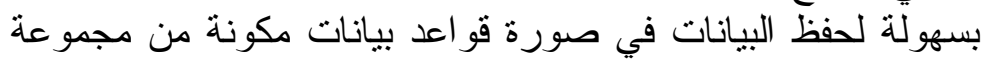

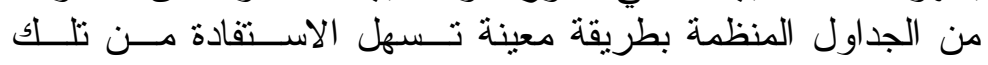

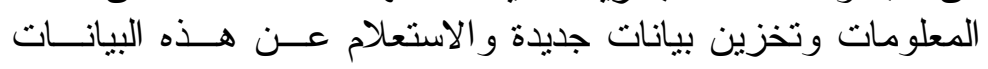

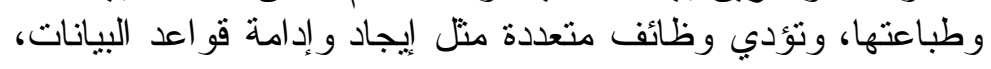

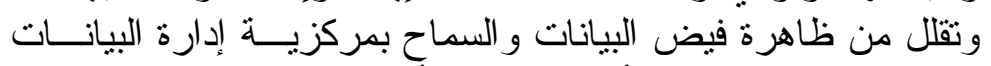

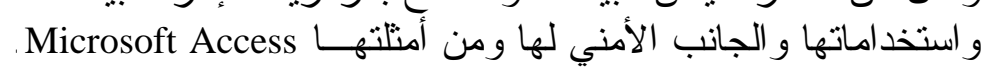

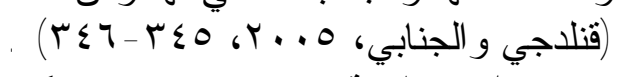

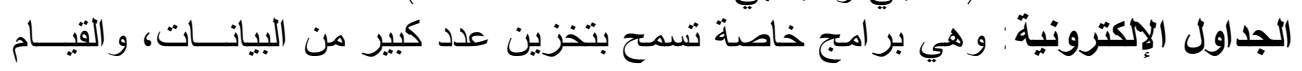

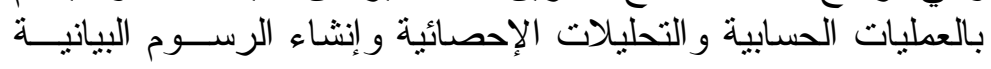




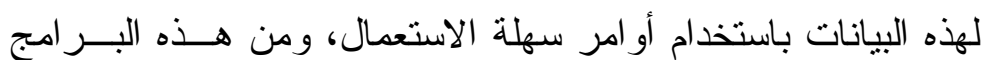

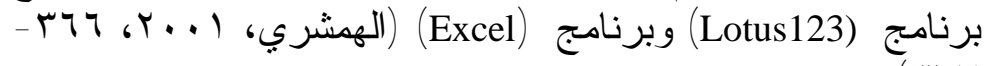

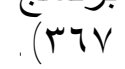

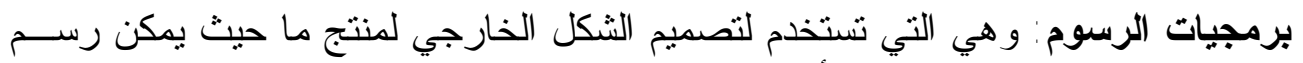

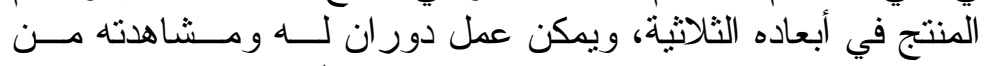

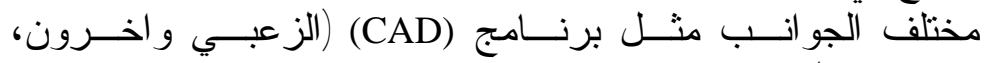
. (Or. r..V

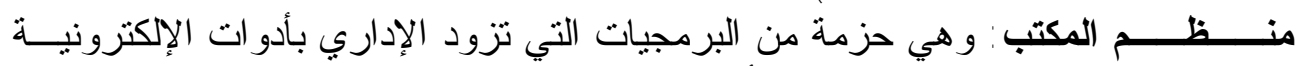

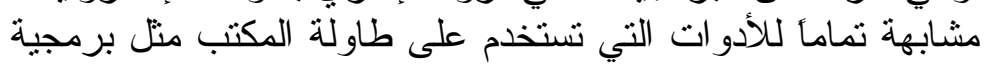

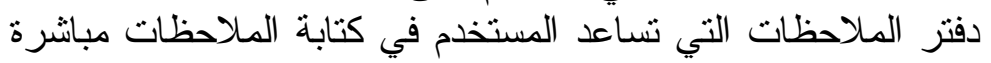

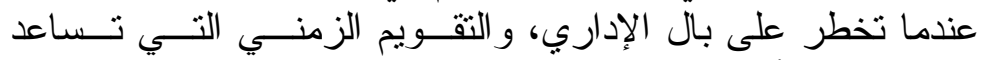

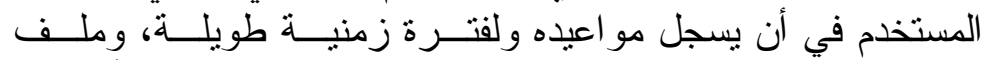

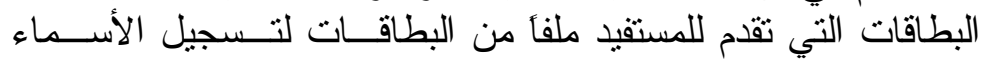

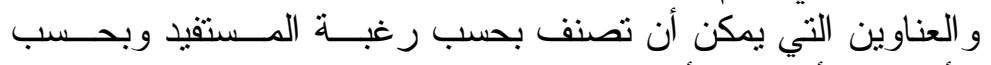

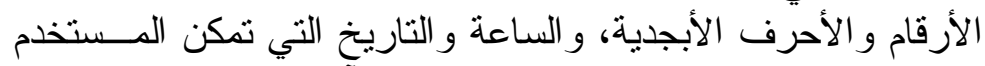

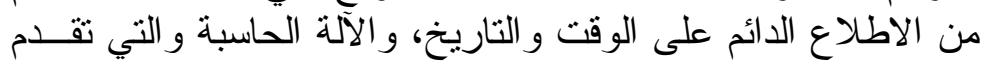

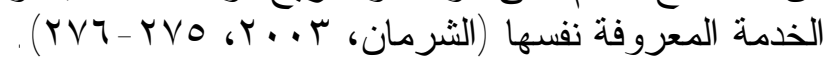

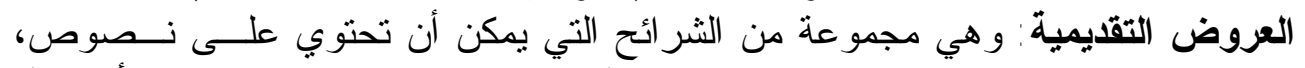

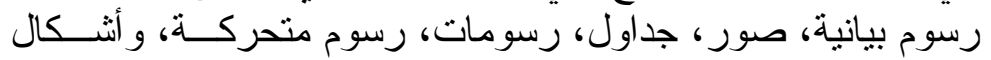

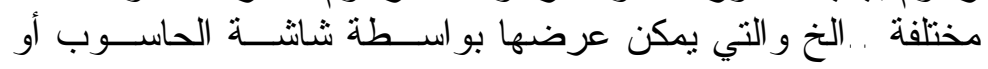

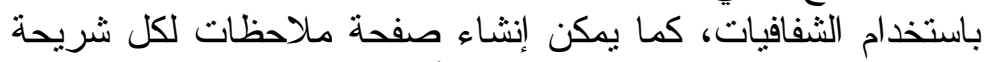

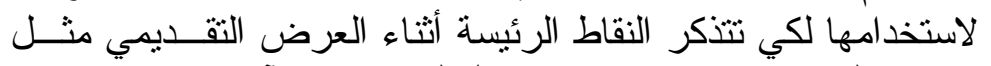

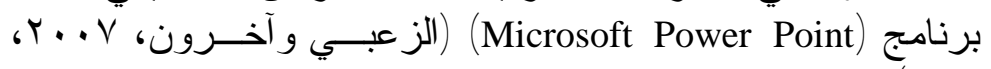
(rvv

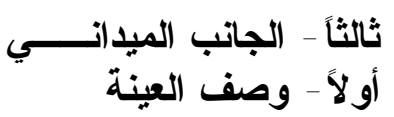

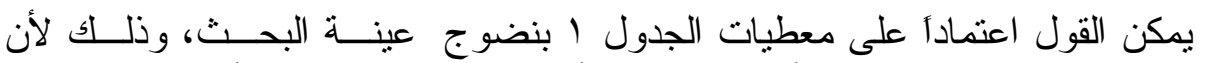

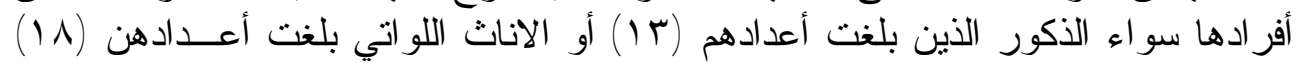

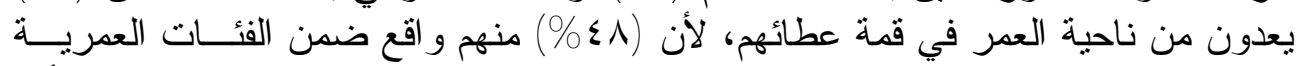

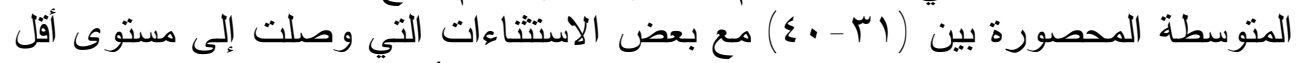

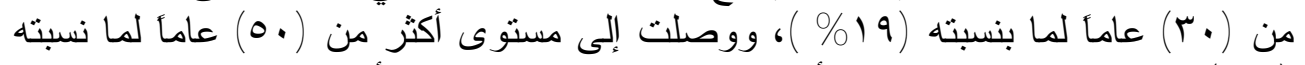

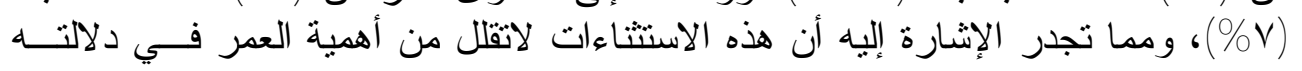

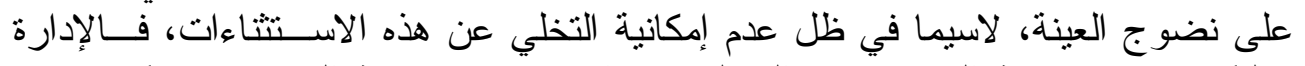

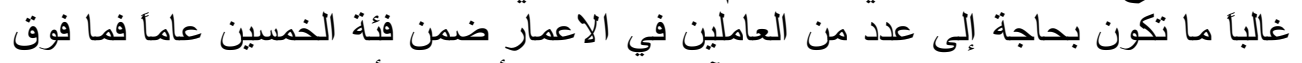

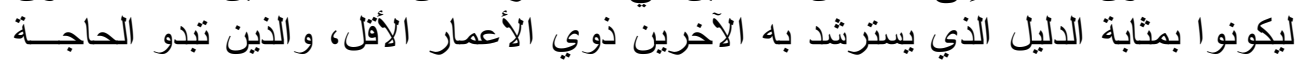

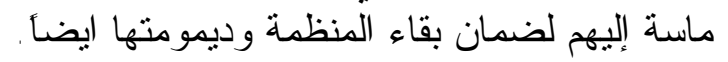




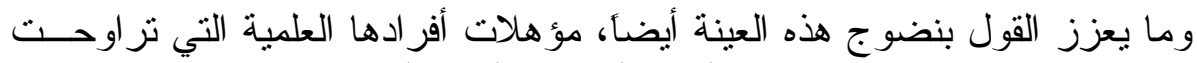

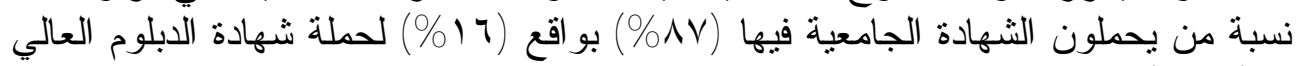

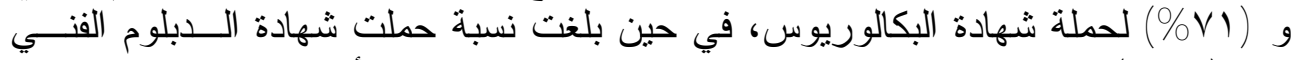

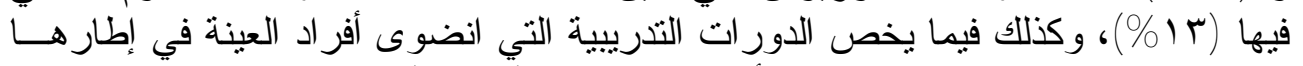

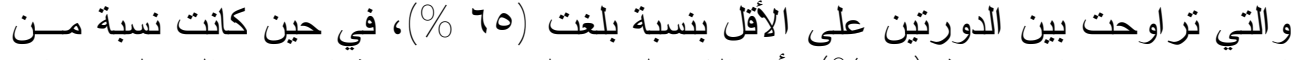

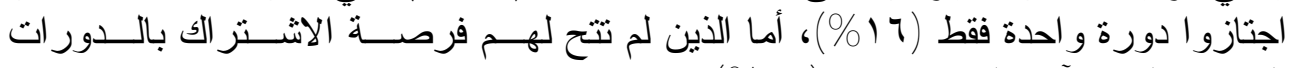

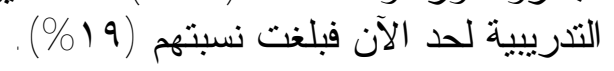

كما ويساعد على القول بنضوج هذه العينة العناوين الوظيفية التي يحملها أفر ادهــا،

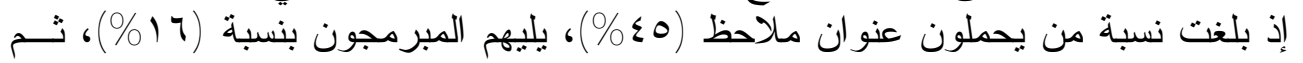

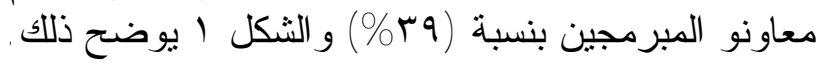

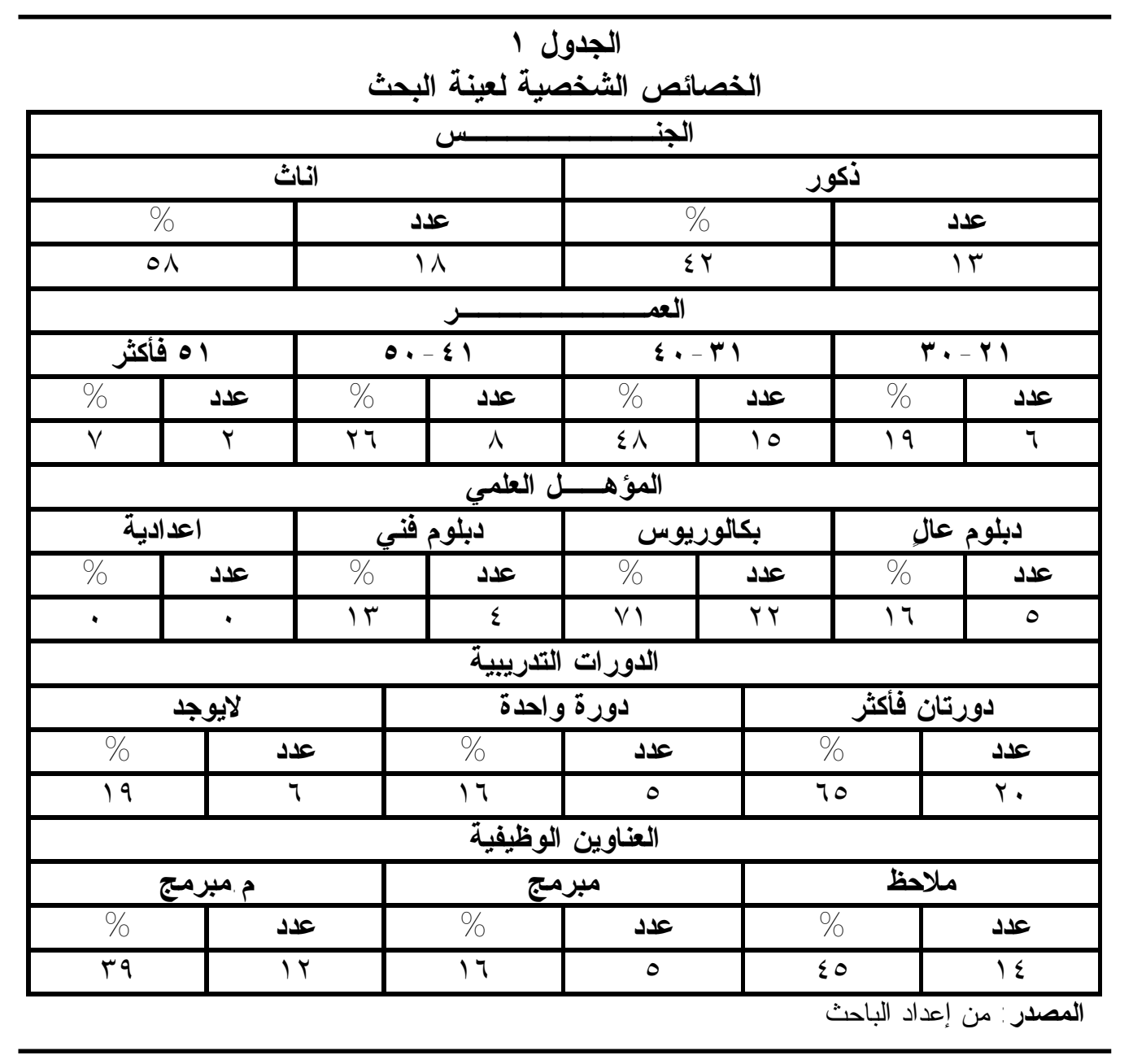




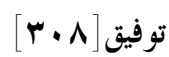

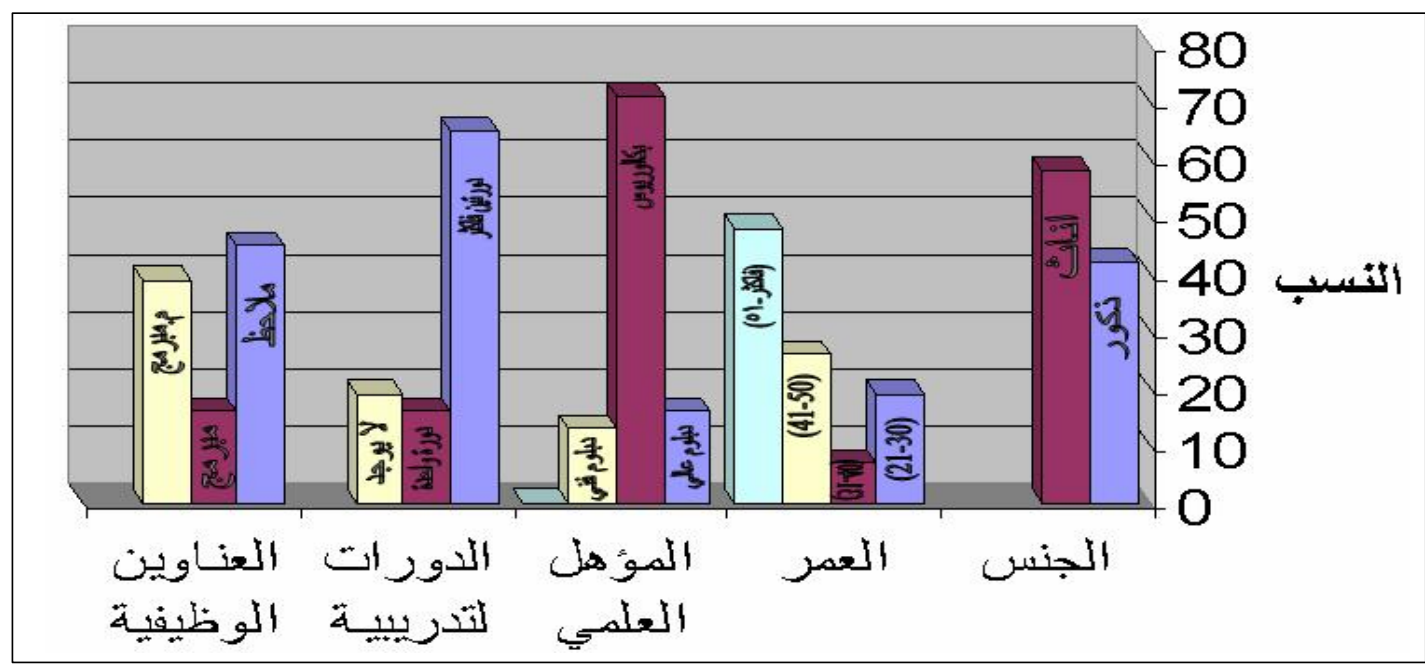

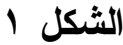

الخصائص الشخصية لعينة البحث

\section{ثانياً - مو اقف المستبينة آرائهم تجاه مؤشرات البحث

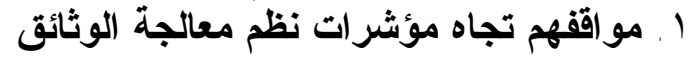

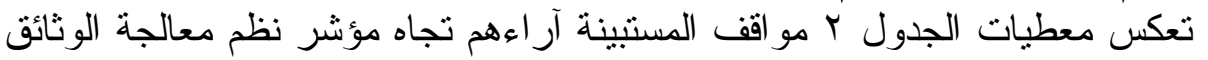

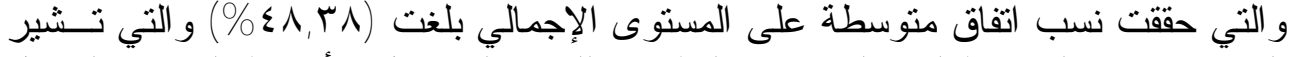

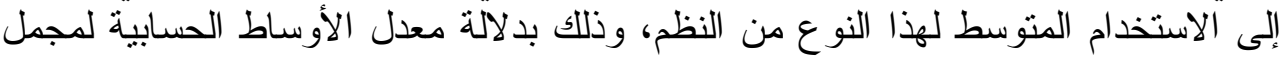

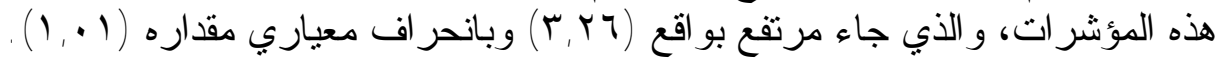

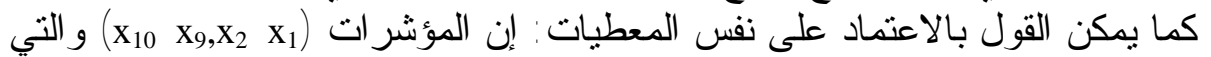

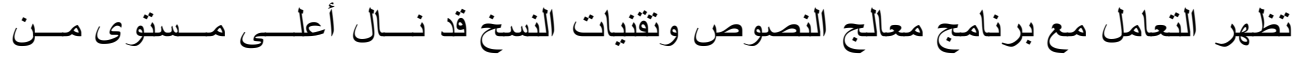

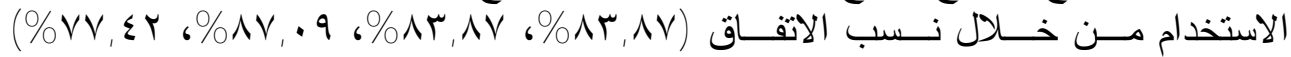

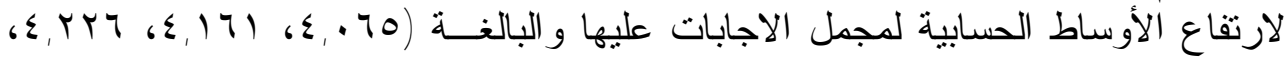

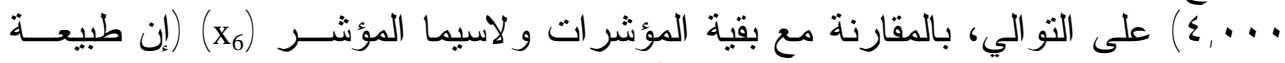

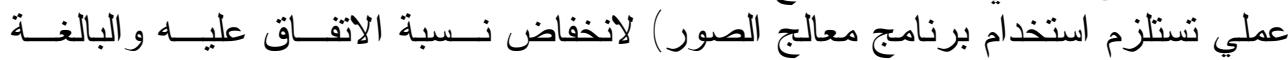

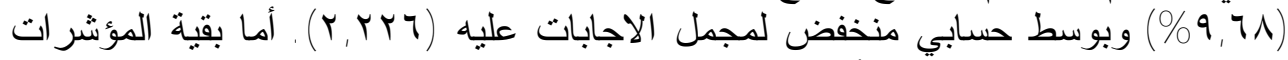

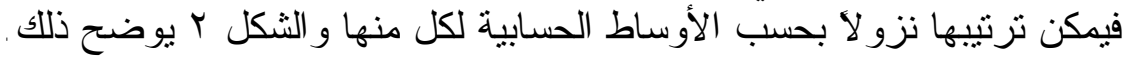




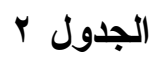

مو اقف المستبينة آراءهم تجاه مؤشر بات نظم معالجة الوثائق

\begin{tabular}{|c|c|c|c|c|c|c|c|c|c|c|c|c|}
\hline \multirow{2}{*}{ المعباري } & \multirow{2}{*}{ الحسابدي } & \multicolumn{2}{|c|}{ منففض جذا } & \multicolumn{2}{|c|}{ منخفض } & \multicolumn{2}{|c|}{ مئوسطظ } & \multicolumn{2}{|c|}{ عائ } & \multicolumn{2}{|c|}{ عالز جداً } & \multirow{2}{*}{ الثؤُسر } \\
\hline & & $\%$ & ت & $\%$ & ت & $\%$ & 5 & $\%$ & ت & $\%$ & ت & \\
\hline - ४หร & $\varepsilon, .40$ & - & - & - & - & $14,14^{\circ}$ & o & Y), ร9 & 19 & YY,OA & $v$ & $x_{1}$ \\
\hline . YरQ & $\{, 1\}$ & - & - & "५. & 1 & १५, q. & $\varepsilon$ & $\varepsilon A, \varphi^{u} q$ & 10 & $Y^{\prime \prime} 0, \varepsilon A$ & $i$ & $\mathrm{x}_{2}$ \\
\hline $1,1 \times 9$ & $r, 0 \leqslant A$ & YY,OA & $v$ & $\Upsilon_{q}, \eta^{\mu}$ & 9 & ५Y,OA & v & $\zeta \zeta, O A$ & $v$ & 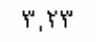 & 1 & $\mathrm{x} 3$ \\
\hline 1,Y̌". & $Y, Y\} Y^{\mu}$ & YY,OA & $v$ & $Y 0, A)$ & A & $Y 0, A\}$ & a & 19,40 & $y$ & $y, \varepsilon 0$ & r & $x_{4}$ \\
\hline$\cdot .99 A$ & ५. १०५" & $\varphi^{\mu,}, \varphi^{\mu}$ & 1 & ५ॅั,ร૫ & 1. & $\varepsilon 1,9 \varepsilon$ & $1 y^{\prime \prime}$ & १५, q. & $\varepsilon$ & $9,9,4$ & $\varphi^{\mu}$ & $x_{5}$ \\
\hline i, is & ร.รัY & $\zeta q_{1} \Upsilon^{\mu}$ & 9 & $\xi^{m} 0, \varepsilon \lambda$ & 11 & $Y O, A\}$ & A & 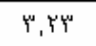 & 1 & 9,80 & r & $x_{6}$ \\
\hline $1, Y A Y "$ & $\Upsilon_{1}, \ldots$ & $A 9,9 A$ & $\xi^{\mu}$ & $\Upsilon^{\prime}, \cdot \varphi_{1}$ & 9 & ५Y,OA & $v$ & $\zeta q_{1} \cdot \eta^{\mu}$ & 9 & $9,9,4$ & $\varphi^{\mu \prime}$ & $x_{7}$ \\
\hline$\cdot .940$ & $r, v \leqslant r$ & 9,91 & $\xi^{\mu}$ & $\Upsilon_{q}, Y^{\mu}$ & 99 & $\varepsilon 1,9 \varepsilon$ & $1 \varphi^{\mu}$ & $14,1 Y^{\mu}$ & o & $Y^{\mu,}, \varphi^{\mu}$ & 1 & $\mathrm{x}_{8}$ \\
\hline., 749 & $\varepsilon$, รY૫ & - & - & - & - & $1 \%, q$. & $\varepsilon$ & 01,11 & 14 & $Y^{\prime \prime O}, \varepsilon A$ & 11 & $x_{9}$ \\
\hline$\cdot A 9 \varepsilon$ & $\varepsilon, \cdots$ & 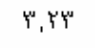 & 1 & - & - & $19,4^{\circ \circ}$ & $y$ & $\varepsilon A, \varphi^{u} q$ & 10 & $\Upsilon_{q}, \zeta^{\mu}$ & 9 & $\mathrm{x}_{10}$ \\
\hline$i, \stackrel{M}{M} \cdot A$ & 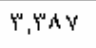 & $1 \%, q$. & $\varepsilon$ & १५.৭. & $\varepsilon$ & $14,14^{\prime \prime}$ & o & $\Psi A, V\}$ & $i r$ & 19,40 & $y$ & $x_{11}$ \\
\hline $1, Y v$. & ५.५৭. & $9,7,4$ & $\xi^{\mu}$ & 19,40 & $y$ & $\zeta \zeta, O A$ & $v$ & 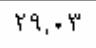 & 9 & 19,40 & $y$ & $\mathrm{x}_{12}$ \\
\hline $1,-1$ & Y, Y૫ & $1 \cdot, r)$ & & in & & 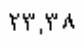 & & $\xi^{\mu} ו, \vee \zeta$ & & $14,7 y$ & & الثبهوع \\
\hline
\end{tabular}

المصدر: من إعداد الباحث

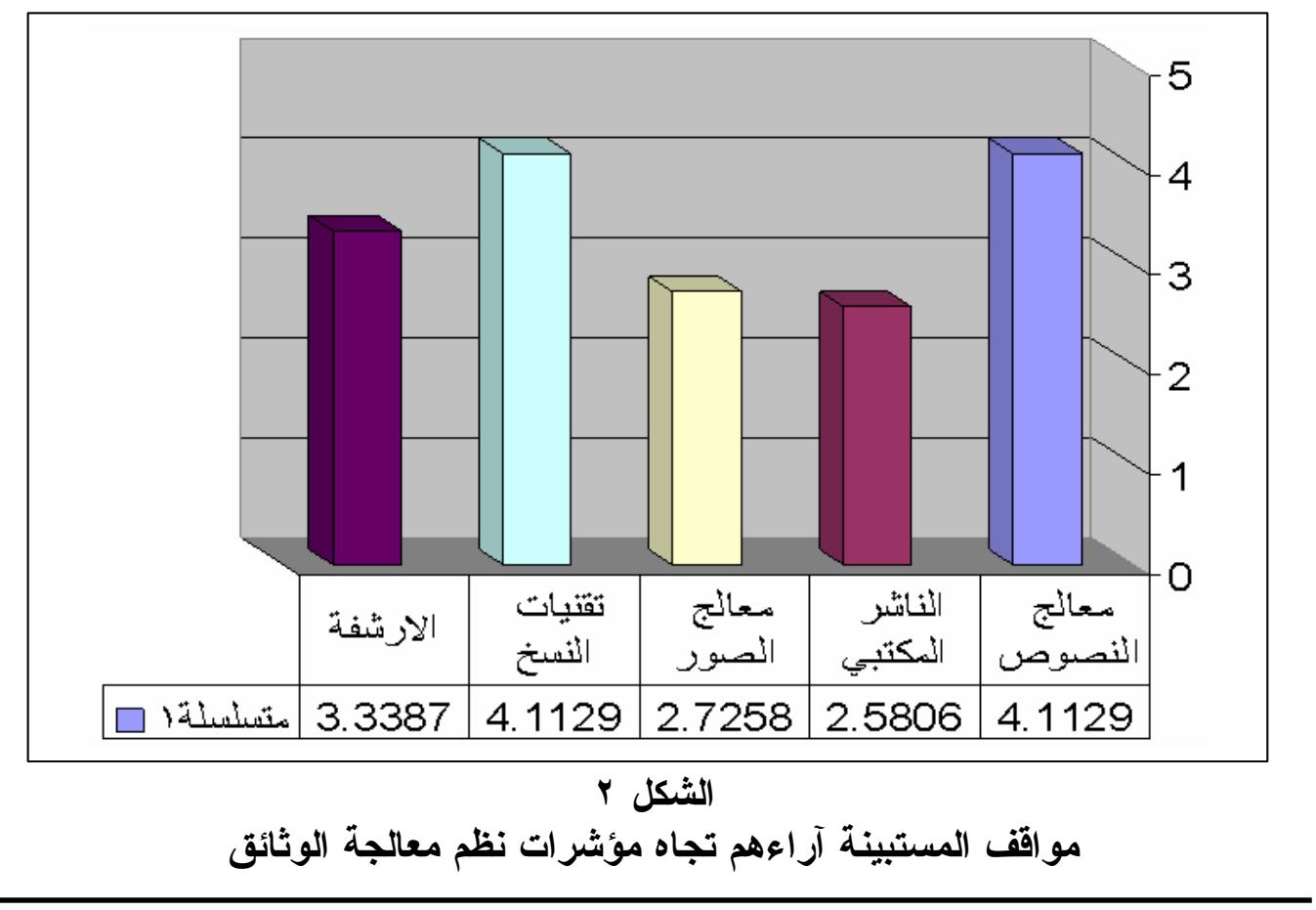




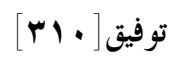

\section{r r مو اقفهم تجاه مؤشر ات نظم الاتصال و المر اسلة}

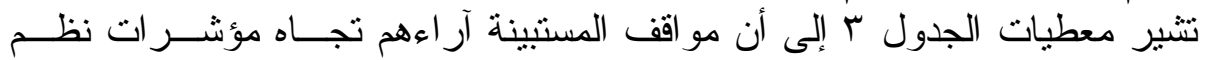

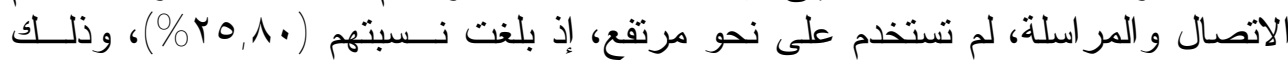

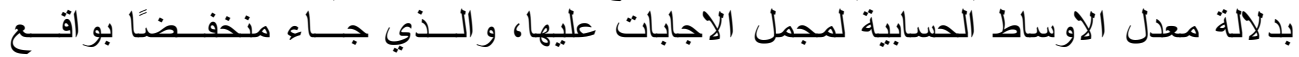

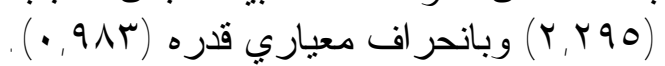

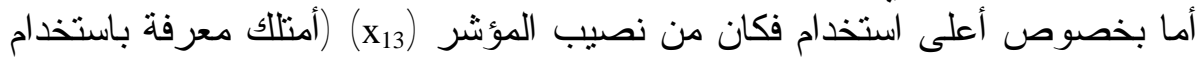

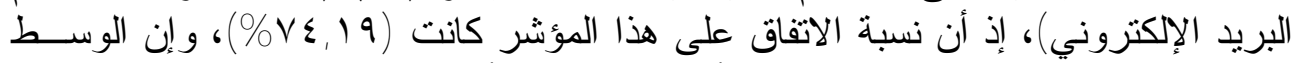

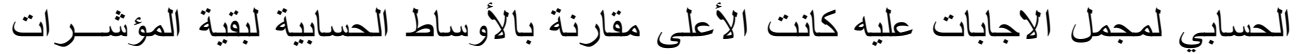

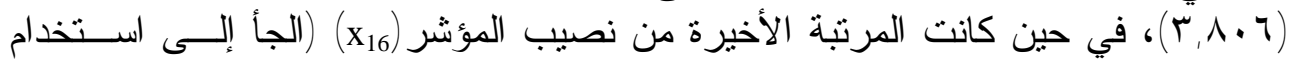

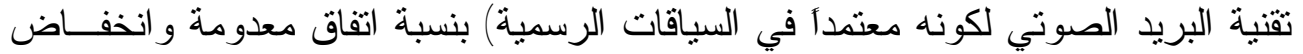

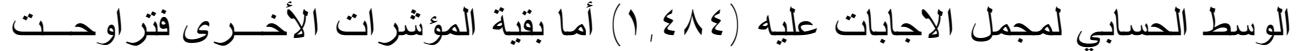

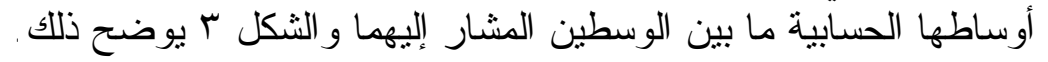

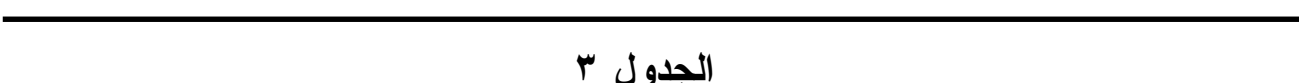

مو اقف المستبينة اراءهم تجاه مؤشرات نظم الاتصال و المر اسلة

\begin{tabular}{|c|c|c|c|c|c|c|c|c|c|c|c|c|}
\hline \multirow{2}{*}{ المعبيار ي } & \multirow{2}{*}{ الثسابوي } & \multicolumn{2}{|c|}{ منخفض جدا } & \multicolumn{2}{|c|}{ منخفض } & \multicolumn{2}{|c|}{ 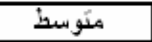 } & \multicolumn{2}{|c|}{ عالز } & \multicolumn{2}{|c|}{ عالز جداً } & \multirow[b]{2}{*}{ الثؤُشر } \\
\hline & & $\%$ & ت & $\%$ & ت & $\%$ & ت & $\%$ & ت & $\%$ & ت & \\
\hline $1,14 \mathrm{~V}$ & $Y^{\prime \prime}, A \cdot Y$ & 4,80 & $\zeta$ & $9,7 \mathrm{~A}$ & $\xi^{\prime \prime}$ & $9,4 \mathrm{~A}$ & $Y^{\mu}$ & 80,14 & $1 \varepsilon$ & ५ด,५५" & 9 & $\mathrm{X}_{13}$ \\
\hline $1, Y$ Yั & $\xi_{1}^{\prime \prime}, 9 v$ & १५.9. & $\varepsilon$ & YY,OA & $\bar{v}$ & १ร.,. & $\varepsilon$ & 80,14 & i६ & 4,80 & $\bar{r}$ & $\overline{X_{14}}$ \\
\hline $1,1 \cdot 1$ & ร.५q. & $\zeta q_{1} \cdot \varphi^{\prime \prime}$ & 9 & $Y Y, Y Y$ & 1. & 19,40 & 4 & 19,40 & $y$ & - & - & $\mathrm{X}_{15}$ \\
\hline$\cdot, v o$ & $1, \varepsilon \wedge \varepsilon$ & $O \varepsilon, A \varepsilon$ & iv & $\varepsilon\}, 9 \varepsilon$ & 1Y" & 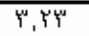 & 1 & - & - & - & - & $\overline{\mathrm{X}_{16}}$ \\
\hline .949 & $1,41 y^{\prime \prime}$ & 41,59 & 19 & $Y 0, A\}$ & $\bar{A}$ & 4,80 & $\bar{r}$ & Y"Y, & 1 & Y", & 1 & $\overline{\mathrm{X}_{17}}$ \\
\hline$\cdot, A 01$ & $1, \varepsilon A \xi$ & $v \cdot, 9 v$ & รัॅ & $1 \%, 9$. & $\varepsilon$ & $1 \%, 9$. & $\varepsilon$ & 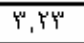 & 1 & - & - & $\overline{X_{18}}$ \\
\hline$\cdot, 9 A Y^{\prime \prime}$ & ५.५९० & $Y^{\mu q} q, Y \varepsilon$ & & Y६, 1q & & 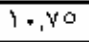 & & 19,90 & & $4, \varepsilon 0$ & & الدجموع \\
\hline
\end{tabular}

المصدر : من إعداد الباحث

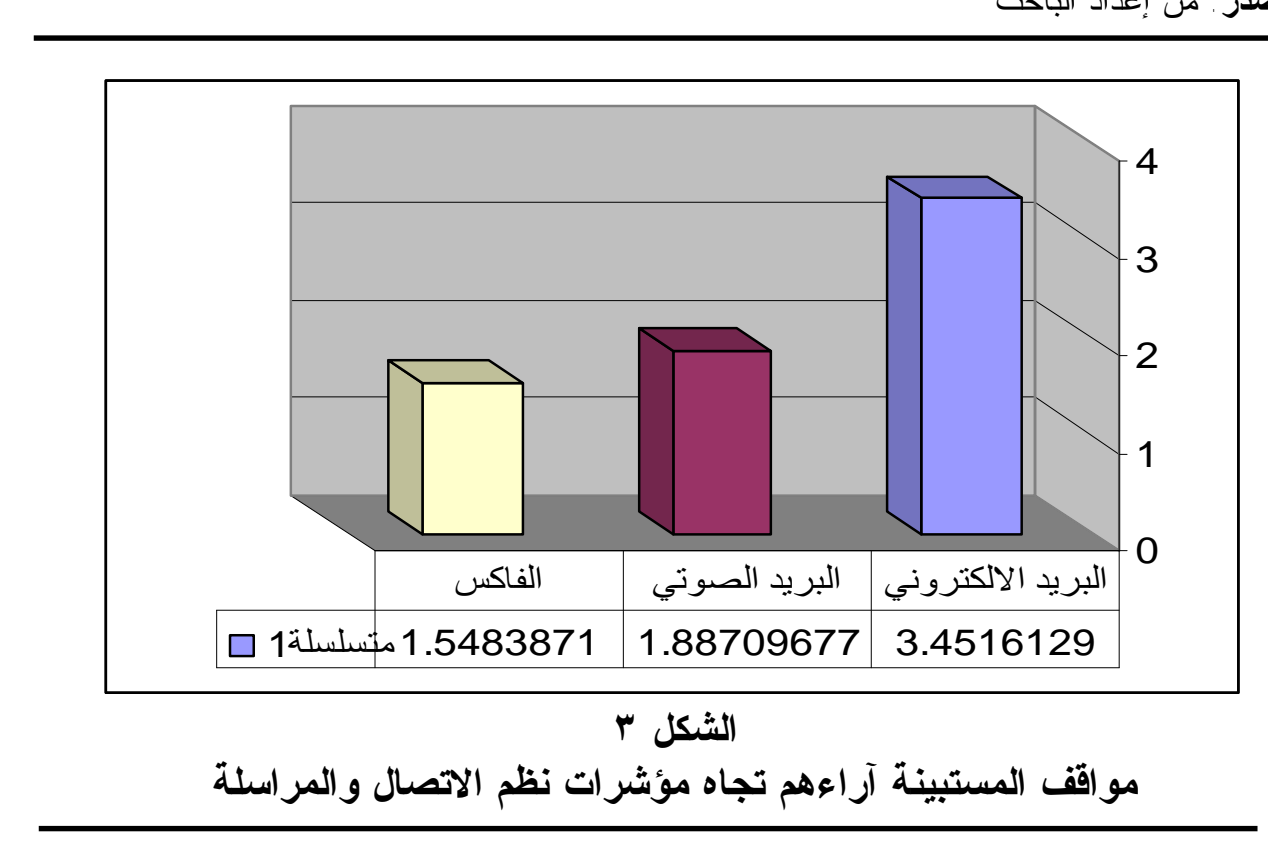




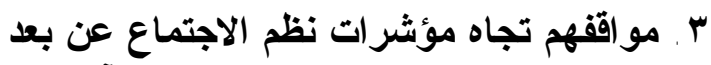

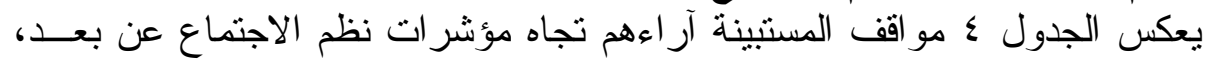

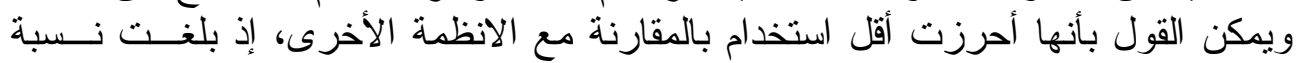

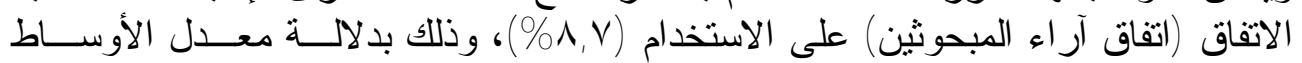

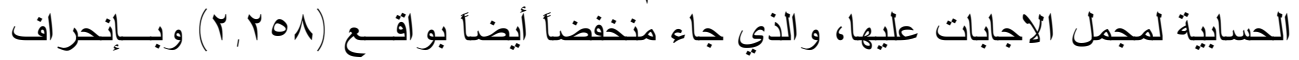

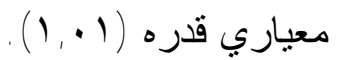

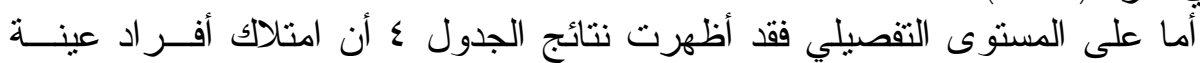

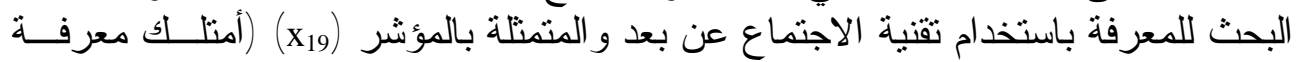

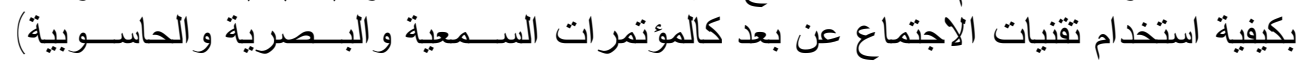

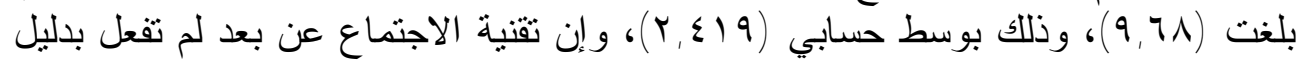

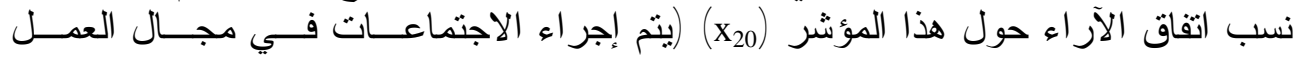

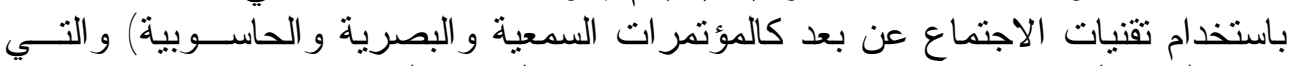

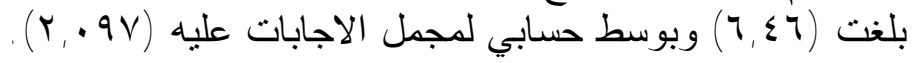

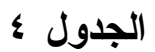

مواقف المستبينة اراعهم تجاه مؤشرات نظم الاجتماع عن بعد

\begin{tabular}{|c|c|c|c|c|c|c|c|c|c|c|c|c|}
\hline \multirow{2}{*}{ 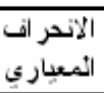 } & \multirow{2}{*}{ أحسابـي } & \multicolumn{2}{|c|}{ منخفض جدا } & \multicolumn{2}{|c|}{ منخفضض } & \multicolumn{2}{|c|}{ مئوسطُ } & \multicolumn{2}{|c|}{ عائي } & \multicolumn{2}{|c|}{ عائي جداً } & \multirow{2}{*}{ المؤشَر } \\
\hline & & $\%$ & ت & $\%$ & ت & $\%$ & ت & $\%$ & ت & $\%$ & ت & \\
\hline$\cdot .99 \%$ & ५.६१९ & $19,9^{\circ 0}$ & $y$ & YैY,YY & 1. & $Y A, Y$ & $i \xi$ & 4,80 & $\zeta$ & 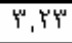 & 1 & $\mathrm{X}_{19}$ \\
\hline $1,0 \leqslant \varepsilon$ & $r_{1} \cdot 9 \gamma$ & $\xi^{\prime \prime O} 0, \varepsilon A$ & 11 & $\zeta q_{1}, \zeta^{\mu}$ & 9 & $\left.\zeta q_{1},\right\}^{\mu \prime}$ & 9 & $\varphi^{\mu,}, Y \varphi^{\mu}$ & $\zeta$ & $Y^{\mu \prime}, Y^{\prime \prime}$ & 1 & $\mathrm{X}_{20}$ \\
\hline 1,01 & ร, צัA & YY, $\varepsilon$ & & $Y^{\mu}=, 4 \varepsilon$ & & $\xi^{\mu \varepsilon} \varepsilon$ & & $\varepsilon, A \xi$ & & $Y^{\mu,}, \xi^{\prime \prime}$ & & الدجهوع \\
\hline
\end{tabular}

المصدر: من إعداد الباحث

ع . مواقفهم تجاه مؤشرات نظم التظيم المكتبي.

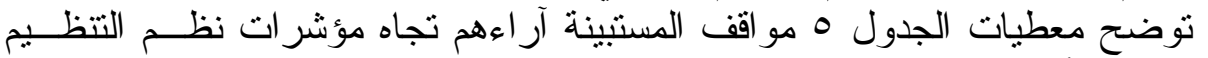

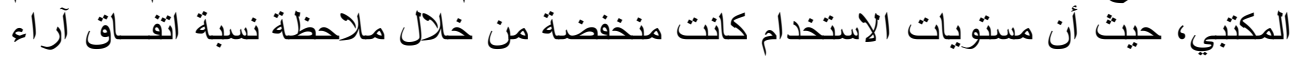
المبحوثين و البالغة (TY,AVY)

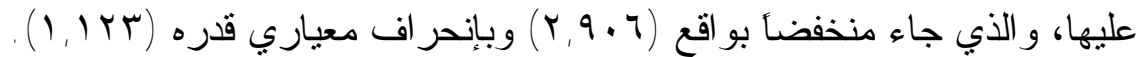

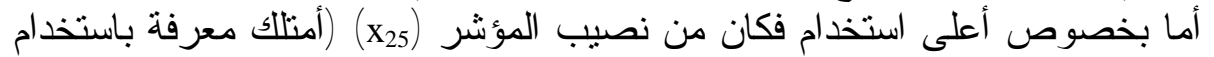

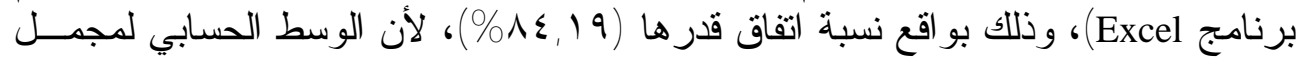

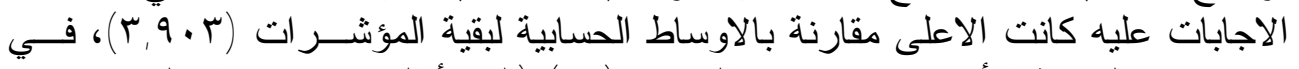

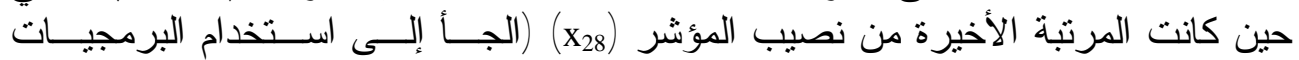

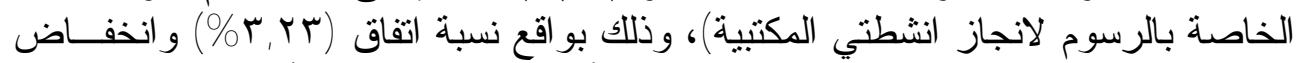

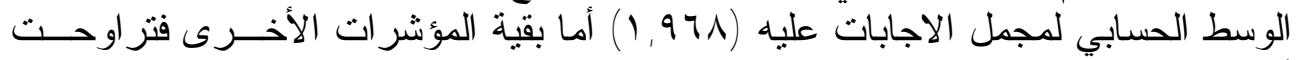

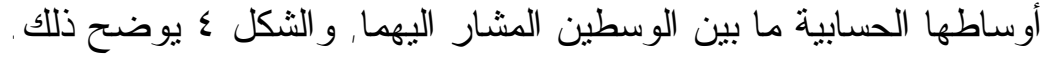




\section{توفيق[r|r]}

\section{- الجدول}

مواقف المستبينة اراءهم تجاه مؤشر ات نظم التظيم المكتبي

\begin{tabular}{|c|c|c|c|c|c|c|c|c|c|c|c|c|}
\hline \multirow{2}{*}{ المعبار ب } & \multirow{2}{*}{ الخسابي } & \multicolumn{2}{|c|}{ منذفض جدا } & \multicolumn{2}{|c|}{ منخفض } & \multicolumn{2}{|c|}{ مئوسط } & \multicolumn{2}{|c|}{ عالز } & \multicolumn{2}{|c|}{ عالز جداً } & \multirow{2}{*}{ المؤُشر } \\
\hline & & $\%$ & ت & $\%$ & ت & $\%$ & ت & $\%$ & ت & $\%$ & ت & \\
\hline$\therefore, 9 \leq \varepsilon$ & $\zeta, q, Y^{\prime \prime}$ & $y, \pm 0$ & $\zeta$ & $\zeta 0, \lambda \uparrow$ & $\lambda$ & $\{1,9 \varepsilon$ & $\pi$ & $\zeta \zeta, O \lambda$ & $\mathrm{v}$ & $r, \zeta \varphi^{2}$ & 1 & $\mathrm{X}_{21}$ \\
\hline $1,51$. & r,A口 & ५५,०А & $\checkmark$ & 14,14 & o & 19,40 & $y$ & $r 0, \leqslant A$ & 11 & $y, \leqslant 0$ & $\zeta$ & $\mathrm{X}_{22}$ \\
\hline 1,1944 & $r, \cdots$ & 14,11 & 0 & ५५,०А & $\mathrm{v}$ & Yo,A & $\lambda$ & 14,11 & o & 19,50 & $y$ & $\mathrm{X}_{23}$ \\
\hline $1, r+1$ & $\zeta, Y \vee \vee$ & $\zeta \zeta, ० \wedge$ & $\mathrm{v}$ & $\zeta q_{1}, r$ & 9 & $19,9$. & $\mathfrak{\xi}$ & $\zeta 9, r^{2}$ & 9 & $4, \leqslant 0$ & $\zeta$ & $\mathrm{X}_{24}$ \\
\hline 1,195 & $r, q, r$ & $r, Y r^{n}$ & $T$ & 4,80 & 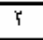 & 14,14 & o & $\{0,14$ & $1 \varepsilon$ & $\zeta q, \varphi^{\prime}$ & 9 & $\overline{X_{25}}$ \\
\hline $1,1 \times \varepsilon$ & $r, 4, r$ & $y, \leqslant 0$ & $\zeta$ & 9,91 & $r$ & $\zeta 0, \lambda)$ & $\wedge$ & Y५,५૫ & 1. & $\zeta \lambda, \lambda)$ & 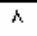 & $\mathrm{X}_{26}$ \\
\hline .919 & $\zeta, 4, Y$ & $y, 60$ & $\zeta$ & $\leq 1,9 \leq$ & $1 \pi$ & $\{1,9 \varepsilon$ & 11 & 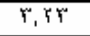 & 1 & $y, \varepsilon 0$ & $r$ & $\mathrm{X}_{27}$ \\
\hline 9,915 & 1,941 & $r \wedge, \nabla$ & $\overline{15}$ & $\zeta q_{1}, \Gamma$ & 9 & $\zeta q_{1}, r$ & 9 & $\Upsilon, \Upsilon \uparrow$ & $T$ & - & - & $\mathrm{X}_{28}$ \\
\hline $1,1,1$ & Y.V. & $15,9$. & $\varepsilon$ & $r Y, Y 4$ & 1. & $r Y, Y Y$ & 1. & 14,11 & a & $y, \leq 0$ & $\zeta$ & $\mathrm{X}_{29}$ \\
\hline 1,155 & Y,Y)Y & 14,14 & o & $50, \lambda)$ & $\wedge$ & $\{1,9 \leqslant$ & $\pi$ & $15,9$. & $\varepsilon$ & $r, \zeta \varphi$ & 1 & $\mathrm{X}_{30}$ \\
\hline $1,51$. & r, ५०A & 14,15 & o & $Y, Y Y$ & 1 & $\zeta q_{1}, \uparrow$ & 9 & $\leq 1,9 \leq$ & $\pi$ & 9,41 & $r$ & $\mathrm{X}_{31}$ \\
\hline $1,51$. & $Y, Y \leqslant Y$ & $\zeta \zeta, \circ \lambda$ & $\bar{v}$ & 14,14 & o & $\zeta q_{1} \varphi^{n}$ & 9 & $\zeta 9, r^{n}$ & 9 & $r^{r}, \Upsilon^{\prime \prime}$ & $T$ & $\mathrm{X}_{32}$ \\
\hline 1,154 & Y,q,4 & $10\{, \lambda 4$. & & $\zeta 1,0, \gamma$ & & $\zeta A, Y Y \varepsilon$ & & ५५,१५० & & $9,9 \leq \mathrm{V}$ & & الهجمبوع \\
\hline
\end{tabular}

المصدر : من إعداد الباحث

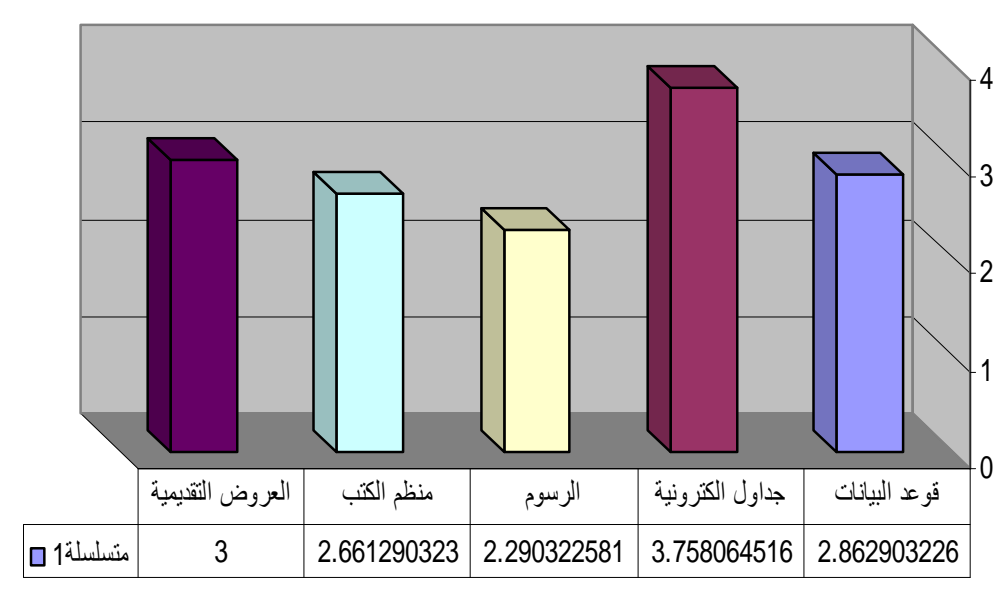

الثكل ع

مواقف المستبينة آراءهم تجاه مؤشرات نظم التظيم المكتبي 


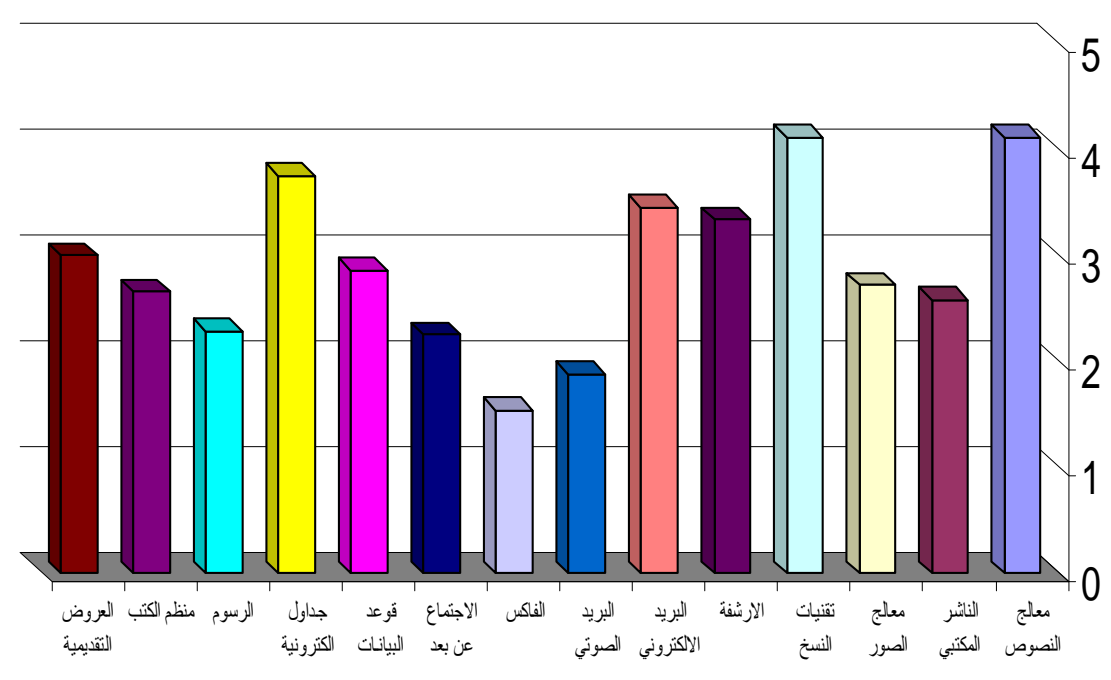

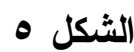

مواقف المستبينة آراءهم تجاه مؤشرات نظم أتمتة المكاتب

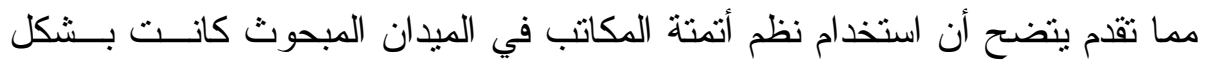

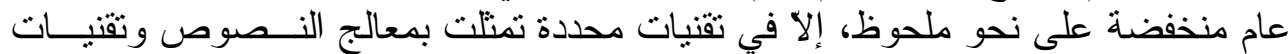

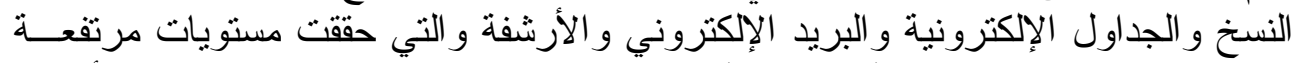

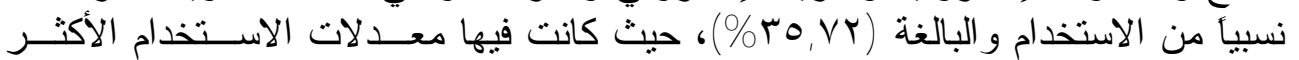

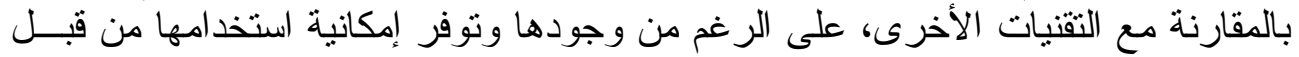

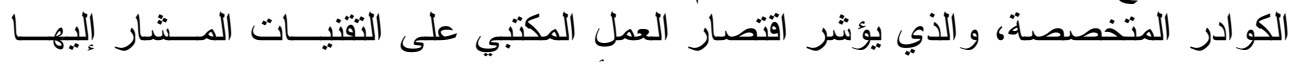

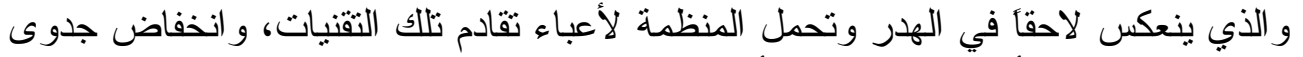

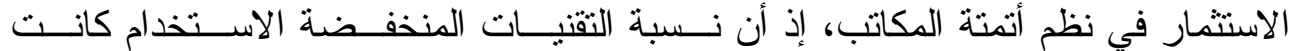

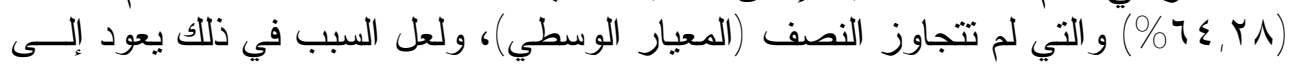

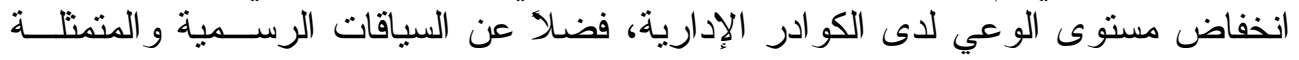

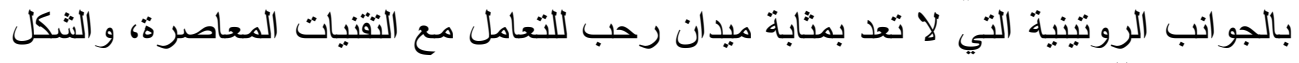
م يوضتح ذللك.

\section{الاستنتاجات والتوصيات.}

الاستنتاجات

ا ـ تعد نظم أتمتة المكاتب أنموذجأ جديدأ للمكاتب يميز المنظمات في القرن الحالي، مــن

$$
\text { هنا فهي تعد : }
$$

أ. مرحلة جديدة من تطور المكاتب المستتدة إلى تقنيات المعلومات بشكل مكثف. 


\section{توفيق[ إ}

ب. متطلباً مهمأ وحيوياً في ظل ثورة المعلومات و الاتصالات التي أدت إلى تغييــر

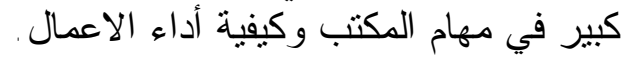

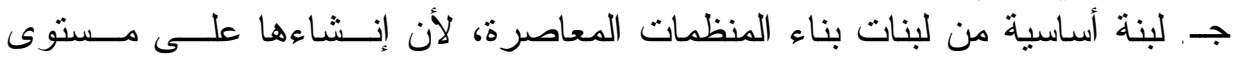
المنظمات يُسهم في تعزيز نظم المعلومات .

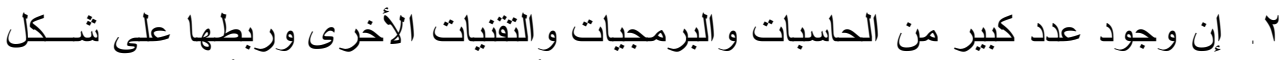
شبكات داخلية وخارجية لايكفي للوصول إلى الأتمتة، و إنما يتطلب الأمر استخدام هذه

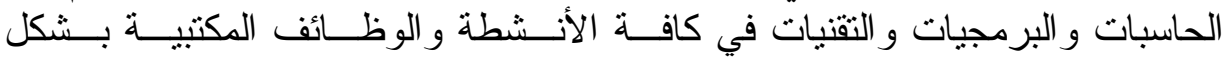
إلكتروني.

r. لم يعد استخدام نظم أتمتة المكاتب خيارَ يخضع للقبول أو الـــرفض، و إنمـــا أصــبح

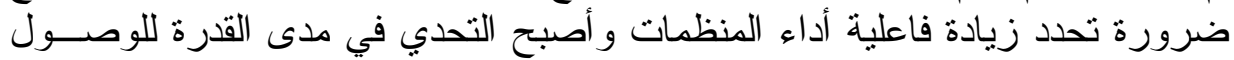

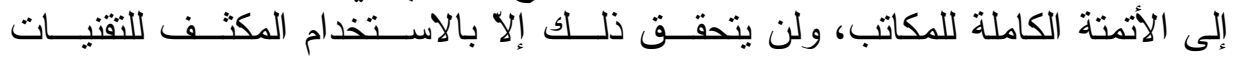

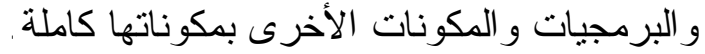

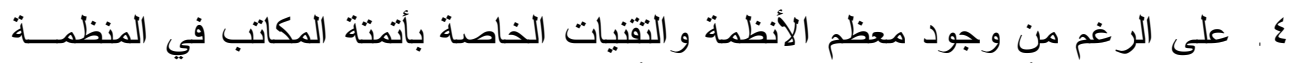

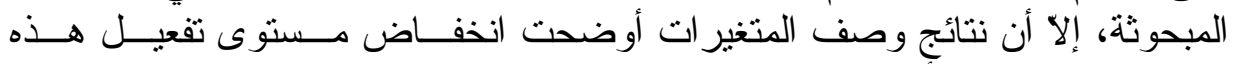
التقنيات في إنجاز الأنشطة المكتبية المختلفة، ولعل السبب في ذلك يعود إلى امــتلاك الكي

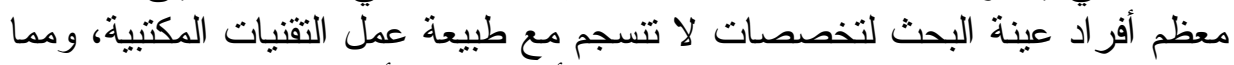

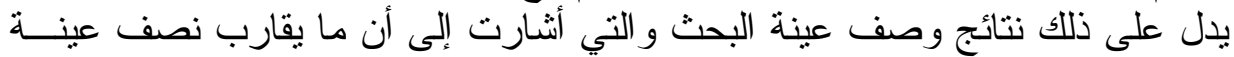
البحث هم من حملة عنو ان الملاحظ.

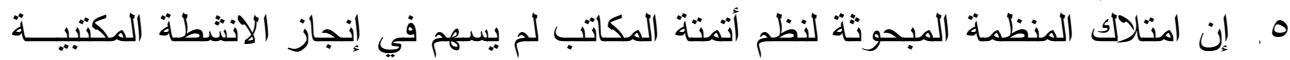

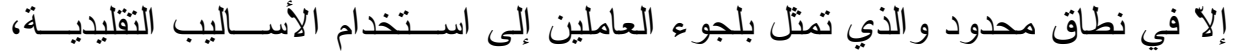

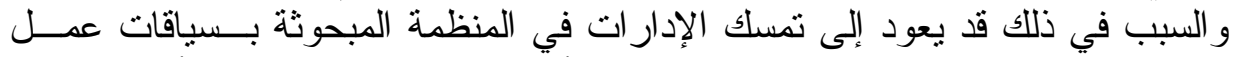

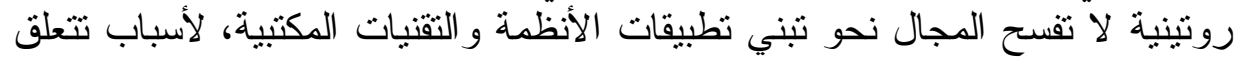

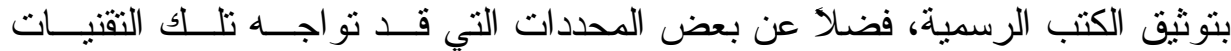

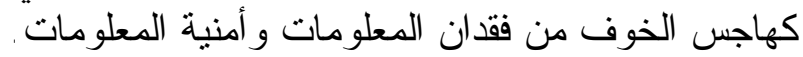

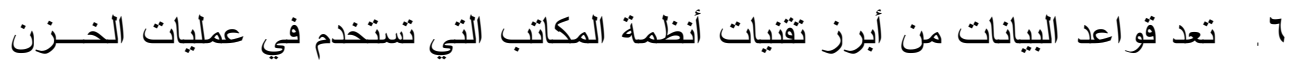

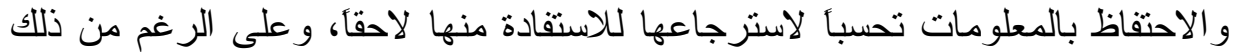

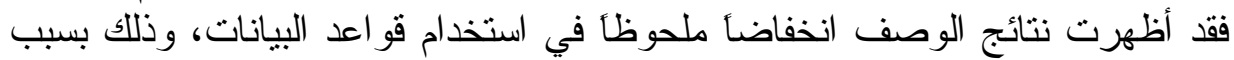

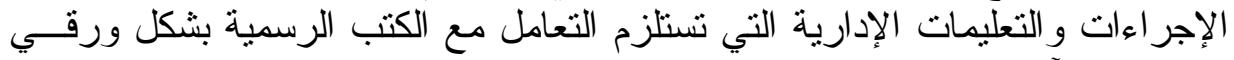
لعدم وجود آلية إدارية وقانونية بالثكل الإلكتروني، فضلا لإدية عن انخفاض مستوى خبرة الكو ادر البشرية.

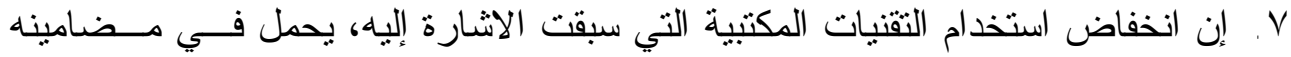

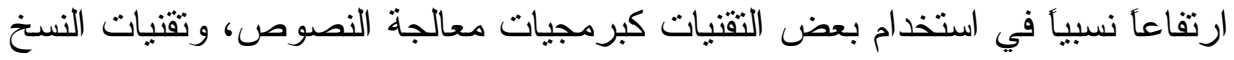

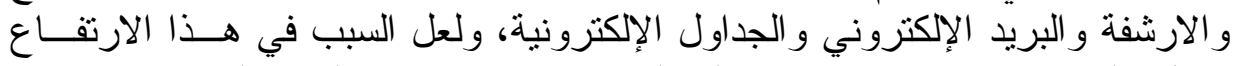

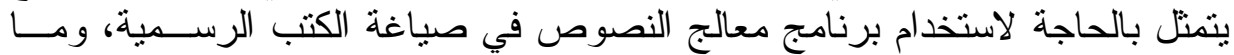

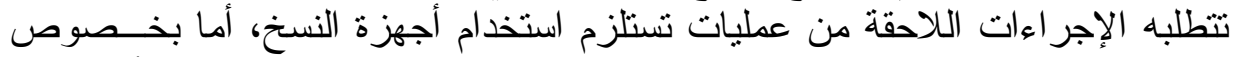

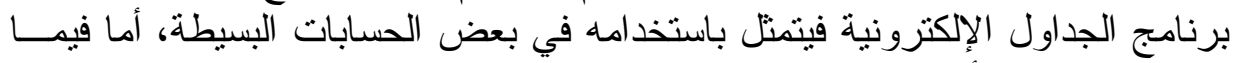

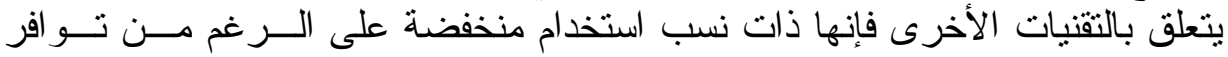
معظمها و إمكانية استخدامها الأنري 


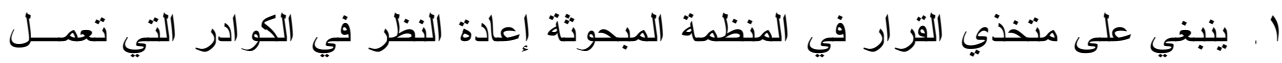

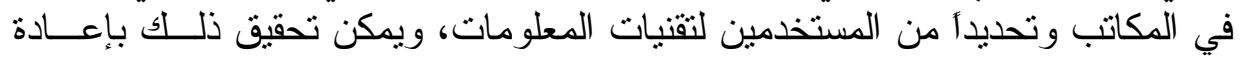

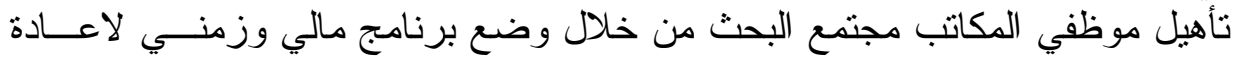

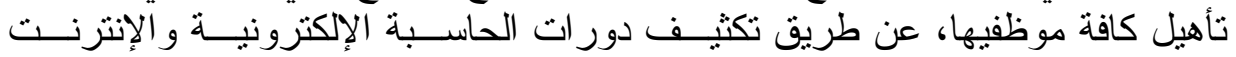

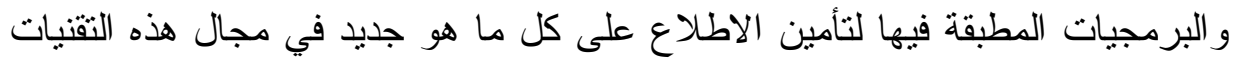

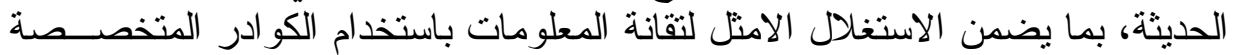
في علوم الحاسبات الإلكترونية.

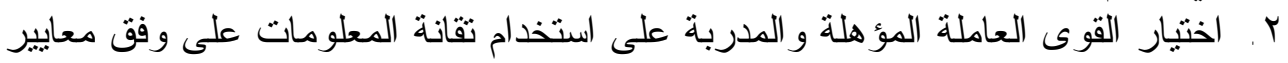

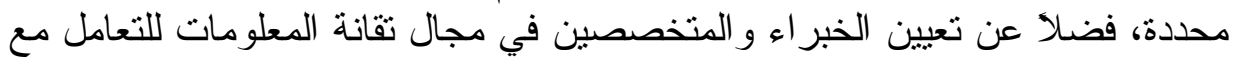
المشكلات التي تو اجه استخدام هذه التئن التقانة.

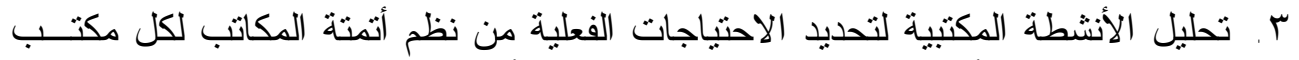

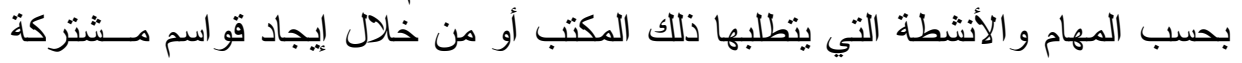

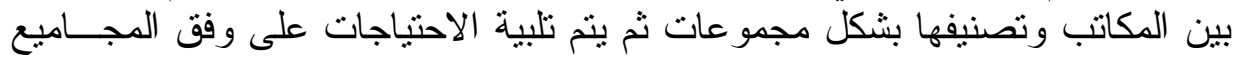

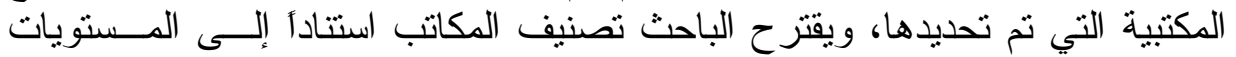
الإدارية.

ع. إن تفعيل استخدام قو اعد البيانات يعد المرتكز الاساسي في الأنظمة الرقمية وبضمنها

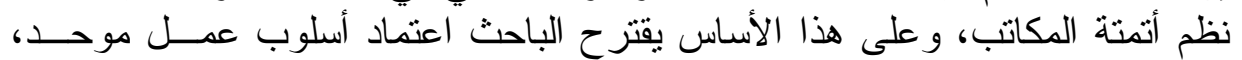

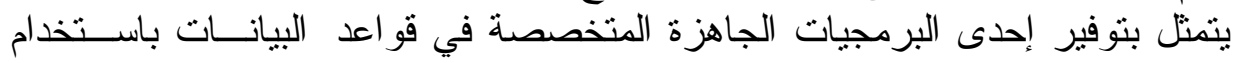

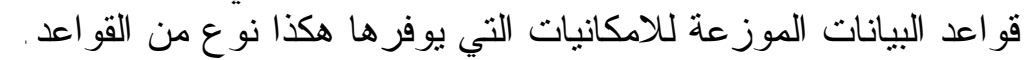

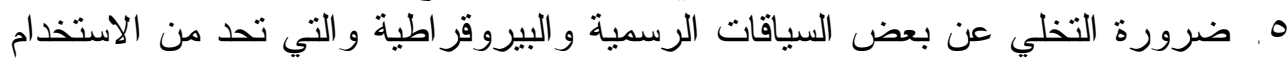

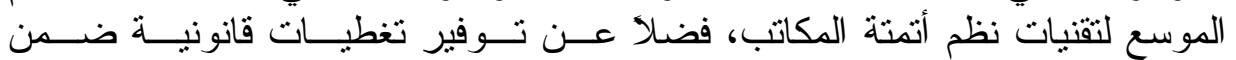
صلاحيات المنظمة المبحوثة للتمكن من حوسبة تلاتك الأنشطة.

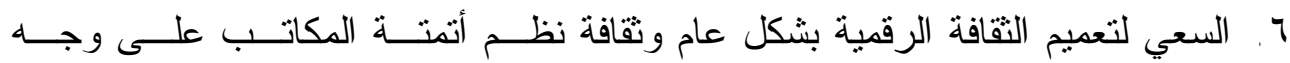

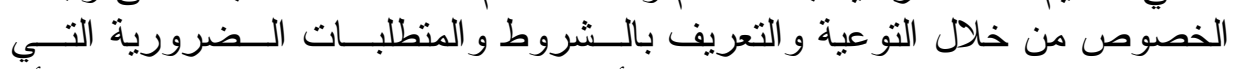

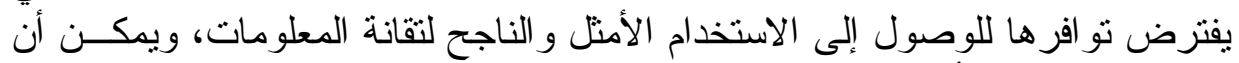

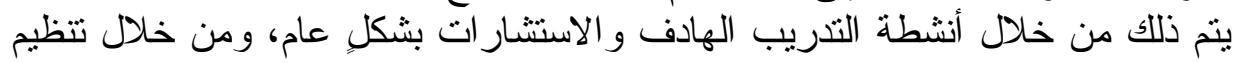
الندو ات و الجلسات العلمية.

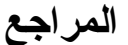

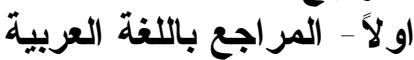

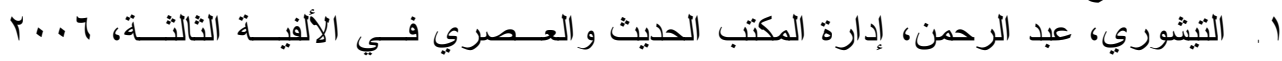
www.ahewar.org/debat/sho

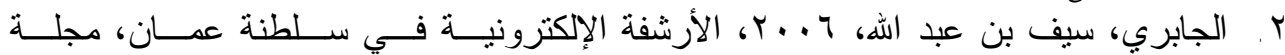

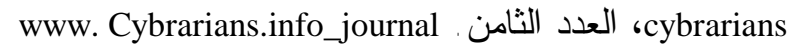

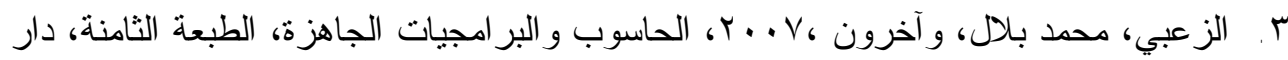

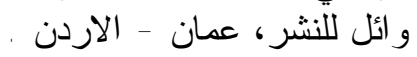

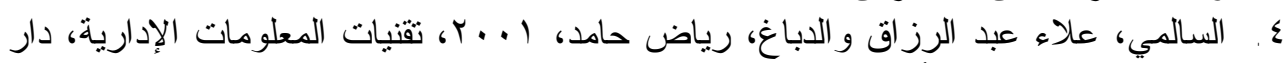

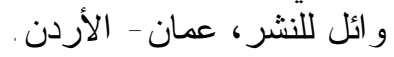




\section{توفيق[1/}

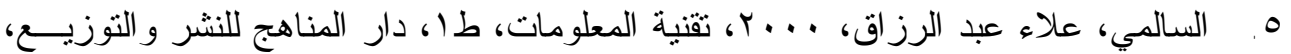

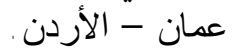

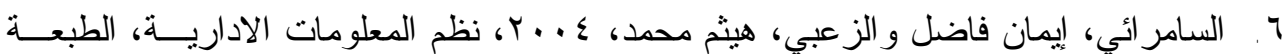

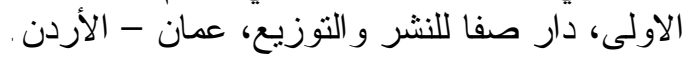

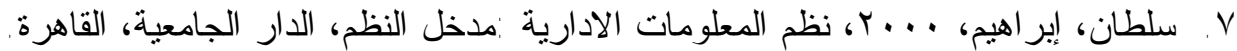

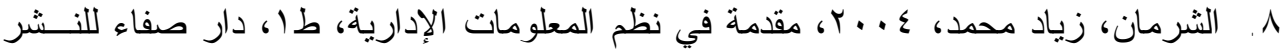

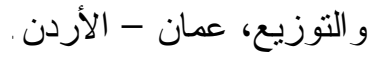

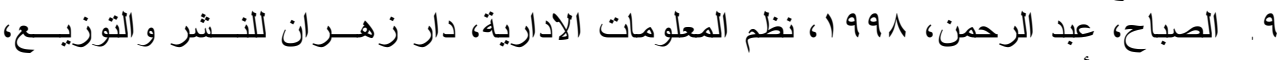

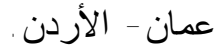

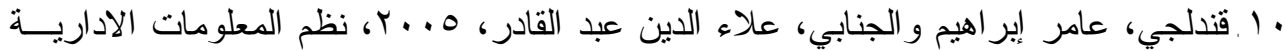

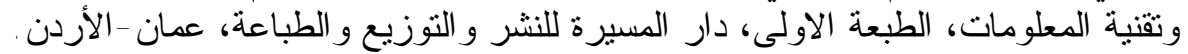

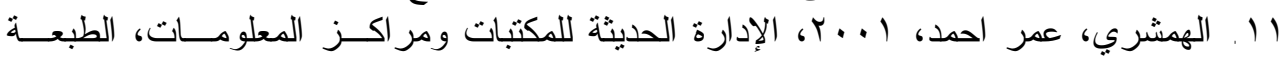

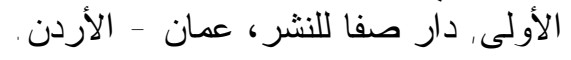

\section{ثانياً - المراجع باللغة الأجنبية}

1. O'Brien, James, (2000), Introduction to Information Systems Essential for the Internet worked Enterprise, $9^{\text {th }}$ edition, McGraw-Hill.

2. www.bookrags.com/research/office-automation-systems-csci-0

1. www.ccasStaft.ps

2. www. faculty.ksu.edu.sa/Al-Arishee

3. www.hazemsakeek.com 